\title{
"NOMINA NUDA TENEMUS"? \\ CONSIDERAZIONI SU FIGURE DIVINE E ICONOGRAFIE A PARTIRE DA ALCUNE TERRECOTTE VOTIVE DELL'AREA SACRA DEL KOTHON A MOZIA
}

\author{
Nicola Chiarenza* - Christian-Albrechts-Universität zu Kiel
}

\author{
"Questi mortali sono proprio divertenti. \\ Noi sappiamo le cose e loro le fanno. \\ Senza di loro mi chiedo che cosa sarebbero \\ i giorni. Che cosa saremmo noi Olimpici [...] \\ Sanno darci dei nomi che ci rivelano a noi stessi". \\ C. Pavese, Dialoghi con Leucò (1947)
}

\begin{abstract}
This paper considers the terracottas discovered in the Sacred Area of the Kothon at Motya during the excavations of the Sapienza University of Rome expedition to Motya in 2005-2008. The analysis of the finds allows us to investigate the possible correlations between the iconographies of the Greektype terracottas and the Phoenician deities that were worshipped in this sacred area. On a further level of analysis this study attempts at looking beyond the names of the characters depicted in the terracottas, by offering some remarks on religious phenomena taking place in Motya as a result of the interaction between different cultural traditions.
\end{abstract}

Keywords: cultural interactions; terracottas; Phoenicians; Greeks; iconography

\section{PREMESSA: LA COROPLASTICA VOTIVA A MOZIA}

Allo stato attuale le testimonianze sulla coroplastica votiva a Mozia sono costituite in maniera predominante dai reperti rinvenuti nel Tofet ${ }^{1}$, cui vanno aggiunti alcuni rinvenimenti ${ }^{2}$ provenienti da contesti cultuali (Santuario del "Cappiddazzu"3, Sacello occidentale antistante Porta Nord ${ }^{4}$, Area Sacra del Kothon ${ }^{5}$, Sacello di Astarte ${ }^{6}$ ), artigianali

\footnotetext{
Desidero ringraziare il Professore Lorenzo Nigro per avermi affidato lo studio di questi reperti, Vorrei ringraziare inoltre il Soprintendente ai BB.CC.AA. di Trapani, Arch. Girolama Fontana e il Dirigente Arch. Antonino Modica per aver autorizzato la pubblicazione delle foto da me realizzate e riprodotte alle figg. 1:f; 4:d. (autorizzazione prot. N. 16275/4 del 15/11/2021 rilasciata dalla Soprintendenza BB.CC.AA. di Trapani). Le restanti foto e i disegni presenti in questo contributo provengono dall'Archivio della Missione Archeologica a Mozia della Sapienza Università di Roma.

1 Sull'argomento si vedano da ultimo Mammina - Toti 2011; 2018.

2 Oltre all'elenco che segue non vanno dimenticati gli esemplari pubblicati in Whitaker 1921, 319-321, figg. 100-101, cui si aggiungano Lagona 2003 e Famà - Toti 2005.

3 Tusa 1964, 38, tav. XXVII; 1973a, 25, tav. XII:2A

4 Isserlin - Coldstream - Snodgrass 1970, 569, fig. 8. La destinazione funzionale di questo piccolo edificio, tuttavia, rimane in parte dubbia (Ciasca 1980, 508-509).

5 Oltre alle terrecotte oggetto di questo contributo, sono da annoverare i seguenti reperti: una testa femminile in terracotta dal riempimento del canale a sud del Kothon (Isserlin 1971, 183, tav. XXX:a), da attribuire verosimilmente ad uno degli edifici sacri dell'area; un frammento di ruota di carro fittile dagli strati superficiali al di sopra del Tempio del Kothon (Nigro ed. 2005, 206, tav. XXXVI:MC.04.40); una matrice per arule raffigurante una sfinge che ghermisce un uomo dal cosiddetto Santuario delle acque (Nigro 2015a, 234, fig. 9); una matrice per protomi femminili (MC.13.100) dal Sacello C7 (Spagnoli 2013, 159; Chiarenza 2015, 53 , nota 19$)$.

$6 \quad$ Nigro 2010a, 167, fig. 13; 2012b, 212, fig. 323.
}

ISSN 0393-0300; 2724-587X

e-ISSN 2532-5159

doi: 10.53131/VO2724-587X2021_2 
(Zona $\mathrm{K}^{7}$ e il cosiddetto "Luogo di arsione" ${ }^{\text {") }}$ ) domestici e/o pubblici (Zona $\mathrm{A}^{9}$, Zona $\mathrm{D}^{10}$, Zona $\mathrm{E}^{11}$, Zona $\mathrm{B}^{12}$, Casa dei mosaici ${ }^{13}$ ).

Ai reperti già noti si aggiungono quelli analizzati in questa sede, ritrovati nell'Area Sacra del Kothon tra il 2005 e il 2008 ad opera dell'Università di Roma "La Sapienza"14 e rappresentati da 12 terrecotte in stato frammentario, riconducibili a diversi edifici sacri all'interno del Temenos Circolare ${ }^{15}$.

\section{LE TERRECOTTE DALL'AREA SACRA DEL KOTHON: ANALISI, CONFRONTI E DATAZIONI}

Nella disamina dei reperti oggetto di questo contributo si seguirà un criterio cronologico, dagli esemplari arcaici a quelli più recenti ${ }^{16}$. Alla descrizione degli oggetti, corredata dall'individuazione dei confronti e dall'attribuzione cronologica ${ }^{17}$, seguiranno le considerazioni sulle produzioni e le iconografie e alcune ipotesi sulle possibili figure divine attestate. Chiude il contributo un catalogo sintetico con le caratteristiche tecniche dei reperti.

7 Falsone 1989, 61, fig. 22 (frammento di matrice raffigurante Eracle e l'idra); Beer 2000 (due protomi femminili); Mammina - Toti 2011, 34, fig. 10 (frammento di matrice per figura femminile "tipo Medma" seduta in trono)

8 Tusa 1973b, 44-45, tav. XXVIII:1-2; 52, tav. XXXV:1; 1978, 72, tav. LIV:3. Non può essere escluso che alcuni di tali reperti, per la loro provenienza da pozzi o fosse, fossero in origine legati a contesti a destinazione diversa da quella produttiva, forse cultuale o funeraria, sconvolti successivamente dall'impianto di installazioni industriali.

9 Famà - Toti - Vecchio 2002, 321-324, nn. 1-3, 7-8.

10 Nigro ed. 2007, 48, 118, tav. IV:MD.03.46, 140, tav. XV:MD.03.338; 152, tav. XXI:MD.04.58, MD.04.85.

11 Una funzione votiva potrebbe essere attribuita alla testa di Sileno proveniente da questo settore dell'isola (Famà - Toti 2000, 456-457, 467, n. 16, tav. XC:1).

12 Toti 2008, 77-78, figg. 8, 10 (matrice raffigurante un sileno con tirso). Nell'edificio denominato "Casa del pozzo quadrato", situato in questo settore dell'isola, sono state rinvenute una testa femminile in terracotta e un'arula raffigurante una sfinge alata (Nigro 2012b, 210, fig. 322).

13 Fiertler 2006

14 I resoconti degli scavi condotti nella Zona C da parte della Missione archeologica dell'Università di Roma "La Sapienza" a partire dal 2002 sono stati pubblicati, oltre che in numerose riviste scientifiche, nei rapport preliminari della serie Quaderni di Archeologia Fenicio Punica: Nigro ed. 2004, 33-138; 2005. Sull'Area sacra del Kothon si vedano da ultimo: Nigro - Spagnoli 2012; 2017, 6-9, 49-56; Nigro 2018, 253-258, 261273.

15 Gli esemplari sono tutti ascrivibili a statuette, protomi o maschere. Non sono stati presi in considerazione in questo contributo i frammenti di arule (MC.06.7, MC.07.126, MC.08.204, MC.08.203, MC.08.96, MC.07.230), lo stampo circolare MC.07.293 e il sostegno MC.07.234, per il quale non può essere assicurata una funzione votiva a causa delle difficoltà di interpretazione dell'oggetto.

16 Nei casi di ampio arco cronologico si terrà conto del termine più alto.

17 La datazione è basata sul contesto di rinvenimento e sui confronti individuati. Anche nei casi in cui questi avrebbero potuto condurre a datazioni al decennio, si sono preferite cronologie più ampie sulla base di due ordini di considerazioni: la frammentarietà dei reperti e la poca affidabilità delle datazioni su base esclusivamente stilistica nei casi di terrecotte prodotte da matrice, in serie e per un lasso di tempo prolungato. $\mathrm{Si}$ vedano a tale proposito le considerazioni espresse da J. Uhlenbrock in merito alle produzioni coroplastiche in Sicilia tra il V e il IV secolo a.C. (Uhlenbrock 2002, 321-326). 


\subsection{Il frammento MC.07.87}

Dall'eschara del sancta sanctorum del Tempio $\mathrm{C} 5^{18}$ proviene la terracotta frammentaria MC.07.87, nella quale le caratteristiche morfologiche e la steccatura della superficie consentono di riconoscere la parte inferiore sinistra di una protome o di una maschera, forse maschile (fig. 1:a). Il frammento è stato attribuito, sulla base del contesto, alla Fase 8 (Motya IVB, 750-675 a.C.) $)^{19}$ e rappresenta pertanto l'esemplare più arcaico fra le protomi e le maschere fino ad ora note da Mozia e uno dei primi fra quelli rinvenuti in ambito fenicio d'occidente ${ }^{20}$. Tenuto conto del contesto di rinvenimento, potrebbe trattarsi di un ex-voto o del simulacro della divinità titolare ${ }^{21}$ del Tempio C5, spezzato ritualmente e deposto nell'eschara.

\subsection{La ruota MC.08.117}

Dalla Zona C Est ${ }^{22}$ proviene la ruota fittile MC.08.117 (fig. 1:b) che costituisce il secondo esemplare di questo tipo rinvenuto nell'Area Sacra del Kothon ${ }^{23}$. Il lato interno della ruota è piano, mentre quello esterno presenta una sporgenza cilindrica dotata di foro passante, all'interno della quale doveva arrestarsi l'asse. La ruota apparteneva ad un carro o ad un quadrupede, oggetti presenti sporadicamente in Sicilia ${ }^{24}$ ma attestati, con numerose varianti, nel corso dell'Età del Ferro nella regione siro-palestinese e soprattutto a Cipro, sia in contesti santuariali che funerari ${ }^{25}$. Il reperto moziese può essere datato tra la seconda metà del VI e la prima metà del V secolo a.C. sulla base dei confronti individuati e del contesto di rinvenimento.

18 Nigro 2010b, 25-26, 32; Nigro - Spagnoli 2017, 49-52.

19 Il sancta sanctorum (L.2110) del Tempio C5 venne realizzato nella Fase 8 (750-675 a.C.) e rimase quasi inalterato nella Fase 7 (675-550 a.C.) quando venne rialzato il piano pavimentale che coprì parzialmente l'eschara della fase precedente (Nigro 2010b, 25-26, 32; 2018, 256).

20 Una delle protomi più arcaiche fino ad ora note da Mozia è una protome femminile di tipo samio, di produzione verosimilmente selinuntina, rinvenuta nel Tofet (Amadasi Guzzo 1969, 59, n. 9, tav. LXIII:1) e databile sulla base dei confronti (Wiederkehr Schuler 2004, 79, 88-90, note 30-31, Type 1B, tavv. 1-2) e del contesto di rinvenimento (base dello strato III) alla seconda metà del VI secolo a.C. (più verosimilmente 540520 a.C. ca.). Ad un arco cronologico leggermente più ampio (tra la seconda metà del VI e i primi decenni del $\mathrm{V}$ secolo a.C.) possono essere attribuite la maschera maschile cosiddetta ghignante e le protomi femminili rinvenute nella favissa centrale dello stesso santuario (Ciasca 1991b, 29-30; Orsingher 2014, 150-152). Per le protomi femminili di Mozia si veda anche la bibliografia citata in nota 32. Per le maschere fenicie e puniche nel Mediterraneo - inclusi gli esemplari più arcaici - si vedano: Ciasca 1988, 354-356; Karageorghis 2004, 414, figg. 276-277; Fariselli 2011; Orsingher 2014, 148, 151; Garbati 2016; Orsingher 2018, 55-60; 2019.

21 Si veda ad esempio il volto maschile in terracotta dal Tofet (Ciasca 1991b, 30-31, fig. 10).

22 Il reperto è stato rinvenuto nella US.2082, tra la navata orientale del Tempio C e il Temenos Circolare.

23 Nigro ed. 2005, 206, tav. XXXVI:MC.04.40.

24 Un cavallino fittile modellato a mano, dotato di ruote simili all'esemplare moziese è stato rinvenuto in Contrada Consi a Butera e datato al VII secolo a.C. (Adamesteanu 1958, 510, fig. 190; Panvini - Sole edd. 2009, 79, n. VII/26). Da una sepoltura della Necropoli del Fusco a Siracusa provengono i resti di un modellino fittile con ruote a cinque raggi datato da E. Woytowitsch $(1978,75$, n. 172, tav. 40:172) per tecnica e decorazione tra l'ultimo quarto dell'VIII e l'ultimo quarto del VII secolo a.C.

25 Per le diverse tipologie di carri fittili si vedano: Karageorghis 1995, 100-123, in particolare p. 104, nn. 3, 5, tav. LV:1 e 3; 1996, 67-72, in particolare n. 21, tav. XXXIX:6. Per alcuni esempi di cavalli con ruote: Karageorghis 1993, 65-67. 


\subsection{Il frammento di statuetta MC.08.127}

Il reperto ${ }^{26}$ (fig. 1:c), proveniente dalla Zona C Sud ${ }^{27}$, è attribuibile alla categoria delle statuette con pettorali ${ }^{28}$, ampiamente diffusa in Sicilia tra la seconda metà del VI e la fine del V secolo a.C. ${ }^{29}$ (fig. 1:d). Fra gli esemplari già noti a Mozia, rinvenuti prevalentemente all'interno del Tofet, alcuni sono prodotti locali ${ }^{30}$, a differenza del frammento in questione che apparteneva a una terracotta di possibile importazione, come testimoniato dall'esame autoptico dell'argilla. Una precisa attribuzione tipologica, con conseguente datazione, è impedita dall'esiguità del frammento, inquadrabile genericamente nei suddetti limiti cronologici di questa classe di terrecotte.

\subsection{La protome MC.08.246}

Il frammento di protome femminile (fig. 1:e), di produzione locale, è stato rinvenuto all'interno del Temenos Circolare, pochi metri a nord-est del cosiddetto Santuario delle Acque Sacre ${ }^{31}$. Si tratta della porzione pertinente all'estremità posteriore della guancia sinistra, dietro la quale si riconoscono $\mathrm{i}$ capelli, raccolti in una treccia resa in maniera stilizzata. La porzione conservata di questa terracotta non rende agevole l'individuazione di un confronto puntuale fra le tipologie di protomi finora note a Mozia ${ }^{32}$, Cartagine ${ }^{33}$ o nei principali centri di produzione sicelioti ${ }^{34}$. Tuttavia, alcuni particolari - quali il tipo di argilla utilizzata, il trattamento superficiale e il modellato della treccia - ricordano la protome

26 La terracotta sembra essere stata realizzata da una matrice priva di nitidezza. Le dimensioni indicano che il frammento apparteneva a una terracotta di grande formato, forse con un altezza complessiva superiore ai 40 $\mathrm{cm}$. Si veda ad esempio la statuetta in Dewailly 1992, 47-48, fig. 13 nella quale le tre file di collane hanno un'altezza totale di $7 \mathrm{~cm}$ e l'altezza della statuetta doveva essere di ca. $50 \mathrm{~cm}$.

27 La terracotta è stata rinvenute nella US.1798, uno scarico di terreno di riporto e materiali realizzato nel IV secolo a.C. contro il Temenos Circolare, nel settore antistante la Porta Sud.

28 Su tale denominazione si veda Pautasso 1996, 46.

29 Su questa categoria di statuette si vedano: Dewailly 1992 (statuette aux parures rinvenute nel Santuario della Malophoros); Albertocchi 1999 (produzioni puniche); 2004; Fiertler 2001 e van Rooijen 2021 (esemplari agrigentini).

30 Per gli esemplari dal Tofet: Albertocchi 1999, 355-356, note 4-6 (con bibliografia precedente); Mammina Toti 2018, 141-146. Una testina femminile in terracotta, di produzione punica, rinvenuta nell'area della casa dei mosaici è stata attribuita a questa categoria e paragonata ad esemplari agrigentini (Fiertler 2006, 11-13, figg. 1-4), mentre un esemplare inedito proviene dalla Zona B ed è esposto nel Museo Whitaker di Mozia (n. inventario MO 999; Fiertler 2006, 12; Mammina - Toti 2018, 146).

31 Il reperto proviene della US.2085, attribuibile alla Fase 4 (Motya VIIA, 470-425 a.C.) e localizzata immediatamente a nord del tratto in cui il muro del Temenos circolare piega ad angolo retto. Sul cosiddetto Santuario delle Acque Sacre: Nigro 2018, 271-273.

32 Sulle protomi rinvenute nel Tofet si vedano Ciasca 1991b, 17-29 e Mammina - Toti 2011, 34-35. A queste vanno aggiunte: una matrice per protomi dal Tempio C7 nel cosiddetto "Santuario delle Acque", pochi metri a sud-ovest della protome MC.08.246 (Chiarenza 2015, 53, nota 19; Spagnoli, 2013, 159; 2017, 102, fig. 8); due protomi dalla Zona K (vedi di seguito); una protome rinvenuta da J. I. S. Whitaker nella necropoli (Famà Toti 2005, 621, fig. 7); un frammento di protome dal Santuario del Cappiddazzu (Tusa 1973a, 25, tav. XII:2A); due protomi frammentarie dal cosiddetto "Luogo di arsione" (Tusa 1973b, 44-45, tav. XXVIII:1B e 2A); una matrice per protomi dall'area di Porta Nord (Chiarenza 2015, 53, nota 19).

33 Per le protomi e le maschere da Cartagine si veda la bibliografia citata in nota 20.

34 Per le protomi da Gela: Uhlenbrock 1988 (una rassegna dei centri di produzione e delle principali attestazioni di protomi in Sicilia, fino al 1988, si trova alle pp. 117-138). A questo si aggiunga Wiederkehr Schuler 2004 per le protomi dal Santuario della Malophoros. 
femminile rinvenuta presso il quartiere artigianale della Zona $\mathrm{K}^{35}$ (fig. 1:f), assimilabile a esemplari selinuntini e caratterizzata su entrambi i lati da una treccia di capelli rigonfia di forma semicilindrica ${ }^{36}$. In quella protome, tuttavia, le ciocche sono rese attraverso incisioni diagonali con andamento irregolare, mentre nella terracotta MC.08.246 sembra potersi riconoscere una treccia "a perle" 37 .

Sulla base di tali osservazioni è possibile proporre che il frammento MC.08.246 appartenesse ad una protome di stile ellenizzante ${ }^{38}$ di produzione locale, simile all'esemplare rinvenuto nella Zona K, ma realizzata da una matrice diversa. Quanto alla cronologia, la terracotta qui esaminata può essere datata nella prima metà del $\mathrm{V}$ secolo a.C., sulla scorta dei confronti proposti e in accordo con il dato stratigrafico.

\subsection{La mano MC.08.210}

Dal versante meridionale del Kothon (US.2354) proviene il frammento MC.08.210 39 raffigurante una mano destra che stringe una melagrana, pertinente con ogni probabilità ad una divinità femminile, forse in posizione seduta (fig. 2:a). Un confronto puntuale può essere individuato nel Santuario della Malophoros di Selinunte, dal quale proviene una terracotta raffigurante una divinità femminile in trono - attribuita agli inizi del $\mathrm{V}$ secolo a.C. - che con la mano destra stringe contro il ventre una melagrana mentre nella sinistra tiene alcuni semi o grani ${ }^{40}$ (fig. 2:b).

Le dee in trono con melagrana sono ricondotte, allo stesso modo delle divinità con patera, colomba o altri attributi, ai tipi di origine locrese-medmea inquadrabili nella prima

35 Beer 2000, 1249-1250, fig.1; la protome è stata datata tra il 500 e il 480 a.C.

36 I volumi rigonfi delle acconciature sono peculiari anche delle protomi di stile greco-fenicio da Mozia (Ciasca 1991b, 21) e sono riscontrabili anche in alcune protomi del Santuario della Malophoros definite di stile punico (Wiederkehr Schuler 2004, 261).

37 Tale particolare stilizzazione dei capelli ricorre in numerose tipologie di protomi selinuntine nelle quali tuttavia si riscontrano sempre due o tre trecce su ciascun lato (Wiederkehr Schuler 2004). La particolarità di una sola treccia "a perle" non sembra invece trovare riscontri precisi. Non può escludersi, tuttavia, che tale caratteristica sia dovuta alla difficile distinzione dei particolari causata dalla generazione della protome da una matrice che aveva perso nitidezza.

38 Quello ellenizzante costituisce uno dei tre stili, oltre a quelle greco-fenicio e a quello egittizzante, proposti da C. Picard nella classificazione delle protomi rinvenute a Cartagine (Picard 1967, 20-29). Tale distinzione è stata adottata da A. Ciasca anche per le protomi di Mozia sulla base della notevole corrispondenza fra gli esemplari moziesi e cartaginesi (Ciasca 1991b, 21; Ciasca - Toti 1994, 9-10). A Mozia, in particolare, le protomi di stile ellenizzante rappresentano il gruppo più numeroso, all'interno del quale gli esemplari realizzati in argille locali da botteghe puniche sono minoritari rispetto alle protomi importate da botteghe siceliote (Ciasca 1991b, 21, 27-28). Ai dieci esemplari appartenenti a questo gruppo rinvenuti all'interno del Tofet vanno aggiunte le due protomi provenienti dalla Zona K (Beer 2000, 1251).

39 Nigro - Spagnoli 2018, 65, fig. 12:e. La terracotta è modellata solo sulla faccia anteriore mentre quella posteriore si presenta concava. L'esemplare è stato realizzato da una matrice che aveva parzialmente perso nitidezza, come dimostra la mancanza di dettagli sull'estremità delle dita e sul frutto. L'assenza del mignolo, invece, sembra attribuibile alle caratteristiche della matrice, che prevedeva una realizzazione sommaria della parte bassa della mano, meno visibile all'osservatore.

40 Gabrici 1927, 273, tav. LX:4. Anche l'esemplare selinuntino è modellato solo sulla faccia anteriore. Per la posizione e il gesto della mano si tratta del confronto più pertinente fra le terrecotte raffiguranti figure femminili stanti o sedute che recano su una mano una melagrana. Per alcuni esempi di figure femminile stanti con melagrana si veda Higgins 1954, 246-247, n. 904 (con amplia bibliografia) 
metà del V secolo a.C. ${ }^{41}$. Questi sono testimoniati a Mozia sia da alcuni esemplari rinvenuti nel Tofet, sia da una matrice dall'area artigianale dell'isola ${ }^{42}$. Sulla base dei confronti il frammento MC.08.210 può essere attribuito allo stesso arco cronologico.

\subsection{La matrice MC.05.396}

La matrice $^{43}$ è stata rinvenuta all'interno del Temenos Circolare, tra questo e il Tempio di Astarte ${ }^{44}$. L'esemplare raffigura in negativo la metà superiore sinistra di un sileno. La nitidezza della matrice, l'accurata caratterizzazione del viso e la realizzazione di un positivo in gesso $^{45}$ (fig. 2:c) consentono di distinguere l'arcata sopracciliare prominente, la palpebra superiore appena rilevata rispetto al maggiore spessore di quella inferiore, il naso dai tratti morbidi sopra i folti baffi. La lunga barba, dal profilo appuntito, resa con sottili linee ondulate è separata, mediante una linea netta e profonda, dallo zigomo prominente; dell'orecchio si riconoscono il profilo esterno arrotondato e la conca al centro del padiglione. La parte conservata del busto è nuda. Il braccio sinistro è aderente al fianco, mentre la mano chiusa impugna l'ansa di un vaso potorio o di un cratere.

L'iconografia del sileno che stringe al petto un kantharos o una coppa, impugnandone le anse, costituisce in ambito coroplastico una delle numerose varianti di sileno accovacciato e sovente itifallico prodotte a partire dal VI secolo a.C. ${ }^{46}$. All'interno di tale gruppo una statuetta conservata a Berlino, sul cui contesto di rinvenimento (verosimilmente in Italia) non si possiedono dati, sembra rappresentare un confronto assai prossimo per la matrice MC.05.396, mentre una terracotta simile potrebbe provenire da Agrigento ${ }^{47}$. Il tema perdura ancora nel corso del IV e del III secolo a.C. sia in ambito punico che italico, come testimoniano una terracotta da Cartagine $^{48}$ e due statuette da Roma ${ }^{49}$, che tuttavia si

41 Su tale tipologia si veda in generale Miller 1983. Per gli esemplari dai contesti sicelioti: Allegro 1990, 128; Bertesago 2009, 61, nota 50

42 Amadasi Guzzo 1969, 71-78, tavv. LXIV-LXV, LXVII. Mammina - Toti 2018, 146-147, figg. 5-6.

43 Una dettagliata analisi iconografica e iconologica è in Chiarenza 2015.

44 Il reperto si trovava nella US.1724a pertinente ad attività di spoliazione del muro del Temenos Circolare (Chiarenza 2015, 51, nota 4). Sul Tempio di Astarte si veda da ultimo Nigro 2019.

45 Desidero esprimere un ringraziamento sentito al Maestro Stefano Ferrari dell'Istituto Centrale per il Restauro di Roma che ha realizzato il calco.

46 Per alcuni esempi si veda Winter 1903a, 216-218. Per le terrecotte raffiguranti sileni itifallici è stata ipotizzata un'origine in area ionica (Blinkenberg 1931, 561-564, nn. 2319-2329, tavv. 108-109) o più in particolare a Rodi (Higgins 1954, 73-74, nn. 159-165, tav. 31; Moscati 1987, 12) intorno alla fine del VI secolo a.C. Un esemplare di tale tipologia (V secolo a.C.), non distante per composizione dalla matrice moziese, proviene dalla tomba 16 della necropoli occidentale di Himera (Vassallo 2005, 79, fig. 127). Nella Sardegna punica due esemplari provengono da Tharros (Higgins 1954, 74, n. 164, tav. 31:164; Moscati 1987, 12-13, tav. I:A1) L'iconografia del sileno con kantharos è attestata nel V secolo a.C. anche su vasi metallici di produzione etrusca (Besques 1982, 269, fig. 9).

47 Per l'esemplare a Berlino si veda Winter 1903a, 218, n. 2 dove viene anche proposto il confronto con la statuetta da Agrigento per la quale, tuttavia, si dispone di una raffigurazione grafica verosimilmente non realistica (Schiavo 1756, 71-75).

48 Cherif 1997, 73-74, n. 231, tav. XXVII.

49 Winter 1903b, 393, n.4; Besques 1982, 269-270, fig. 10. 
distinguono dalla matrice MC.05.396 per l'iconografia del volto e la maggiore prossimità del vaso alla bocca ${ }^{50}$.

I confronti più pertinenti per il viso del sileno raffigurato nella matrice sono individuabili, tuttavia, in un'altra classe di reperti fittili: le antefisse sileniche di Tipo B della classificazione realizzata da P. Pelagatti, inquadrabili nel V secolo a.C. ${ }^{51}$. Tali produzioni influenzarono verosimilmente anche un altro oggetto in terracotta da Mozia, il quale a sua volta presenta alcune analogie con la matrice MC.05.396. Si tratta di un oggetto di incerta funzione - forse un sostegno - che raffigura la testa di un sileno realizzata a stampo, con ritocchi a stecca e a mano ${ }^{52}$. La terracotta, di produzione moziese, accomuna l'iconografia silenica di tradizione greca con alcuni particolari iconografici e tecnici della coroplastica punica ed è stata datata agli inizi del V secolo a.C.

Tali considerazioni inducono a proporre per la matrice MC.05.396 una datazione nel V secolo a.C., forse nel corso della prima metà.

\subsection{La base MC.08.225}

Il frammento di base con piede destro, denominato MC.08.225 (fig. 2:d), è stato rinvenuto nel settore nord-ovest del Temenos Circolare, nei pressi del tratto in cui il muro piega ad angolo retto. La terracotta, di produzione locale, apparteneva, verosimilmente, ad una figura femminile stante con lunga veste. Tale osservazione si basa sull'orlo inferiore dell'abito, sulla ridotta lunghezza della base - incompatibile con la profondità delle basi delle figure femminili in trono - e sull'assenza di caratterizzazione della parte posteriore del piede, le cui dita sono appena distinguibili probabilmente a causa della perdita di nitidezza della matrice ${ }^{53}$.

Frammenti analoghi, per morfologia e dimensioni, provengono da uno scarico di materiale votivo realizzato nel settore nord-orientale del Tofet ${ }^{54} \mathrm{e}$ da uno dei pozzi dell'area

50 La presenza del kantharos accostato alla bocca contraddistingue anche un particolare categoria di statuette (interpretate come thymiateria) nota con il termine di sileno-fiore, nelle quali l'iconografia del personaggio mitologico è differente da quella dei secoli precedenti; alcuni esemplari databili al III secolo a.C. provengono da Selinunte (Cavallari 1884, 323, tav. V:441 e 489; Bisi 1968, 44, figg. 6-7), Morgantina (Bell 1981, 233, n. 932, tav. 138:932), Capua (Besques 1982, 268, figg. 6-8; l'attribuzione alla tipologia dei sileni-fiore di questa terracotta -ritenuta dall'autore una comune statuetta- si basa sulla presenza di un foro circolare sulla sommità della testa, verosimilmente destinato ad alloggiare il calice del fiore).

51 Pelagatti 1965. All'interno del Tipo B, che si discosta dal tipo A per la perdita dei tratti ferini del sileno, la variante $\mathrm{B} 3$ presenta le analogie più stringenti con il volto raffigurato sulla matrice moziese come dimostra in particolare un esemplare da Naxos (Epifanio 1990, 104, 254-255, n. 91). Si veda inoltre un esemplare frammentario da Camarina (Pelagatti 2003, 518, fig. 4). Sono state sottolineate analogie formali e stilistiche tra le antefisse sileniche di Naxos e le serie monetali con Dioniso e il sileno, coniate dalla stessa colonia nella prima metà del V secolo a.C. (da ultimo Albertocchi 2012, 157). Sul rovescio dei tetradrammi appartenenti a questa serie è raffigurato frontalmente un sileno itifallico accosciato che impugna con la mano sinistra un kantharos (Garraffo 1990, 155-156).

52 Chiarenza 2015, 53, fig. 6 (con bibliografia precedente).

53 L'orlo inferiore della veste che copre il piede è privo di caratterizzazione ma tale particolare non è dirimente per una più puntuale determinazione del tipo di abito, poiché anche nei casi di divinità in trono o di korai stanti vestite da un lungo chitone a fitte pieghe, il lembo inferiore della veste appare spesso privo di caratterizzazioni. Si veda ad es. la terracotta citata in nota 55.

54 Amadasi Guzzo 1969, 93, n.104, tav. LXVII:7. 
artigianale nota come "Luogo di arsione" di attribuirla ad un determinato tipo all'interno delle produzioni di figure femminile stanti ${ }^{56}$, la base MC.08.225 può essere genericamente datata al V secolo a.C.

\subsection{La testa MC.08.233}

La terracotta, rinvenuta nell'area antistante la Porta $\mathrm{Sud}^{57}$, raffigura la metà anteriore di una testa femminile di produzione non locale (fig. 3:a). L'esiguità del frammento non consente di ricostruire il resto della figura anche se la resa di alcuni particolari del volto come le labbra e, soprattutto, l'inizio della capigliatura con ondulazioni parallele a chevrons sono caratteristiche peculiari di tipi femminili prodotti a partire dalla fine del primo quarto del V secolo a.C. ${ }^{58}$ (fig. 3:b). Il perdurare delle iconografie, di cui proprio tale tipologia di statuette testimonia un caso eclatante ${ }^{59}$, induce a proporre per questo frammento, realizzato da una matrice che aveva perso nitidezza, una datazione più ampia che va dal secondo quarto alla fine del $\mathrm{V}$ secolo a.C.

\subsection{Il panneggio MC.06.80}

In questo frammento, proveniente dalla Zona $\mathrm{C} \mathrm{Nord}{ }^{60}$, è possibile riconoscere il fianco sinistro di una figura femminile stante, forse il tratto in cui l'himation si sovrappone al chiton con delle pieghe semicircolari progressivamente più alte verso il centro della figura (fig. 3:c). La particolare conformazione del lembo dell'himation consente di accostarlo ad alcune peplophoroi ${ }^{61}$, sebbene sulla base dell'assenza del braccio disteso - che solitamente in questa classe di statuette fiancheggia il tratto in cui i due indumenti si sovrappongono si potrebbe riconoscere una figura con il braccio sinistro levato all'altezza del petto ${ }^{62}$; l'ipotesi sembra essere supportata dal leggero rigonfiamento della superficie sull'estremità superiore del frammento, forse dovuto all'originaria presenza del braccio.

55 Tusa 1973b, 52, tav. XXV:1. Nell'esemplare, attribuito agli inizi del V secolo a.C. e di produzione non locale, il personaggio veste un lungo chitone a fitte pieghe le quali, tuttavia, sono assenti dall'orlo inferiore della veste che ricade sopra il piede.

56 In generale su tale argomento si vedano: Allegro 1990, 124-129; per gli esemplari dal Santuario della Malophoros presso Selinunte e dal Santuario di Bitalemi presso Gela: Bertesago 2009, 60-61 (con bibliografia precedente); per gli esemplari da Morgantina: Bell 1981, 123-124, tavv. 3-4. Per la particolare tipologia di statuette con porcellino si veda Sguaitamatti 1984

57 Il reperto proviene dalla US.2452, uno scarico di terra di riporto e materiali pertinenti all'area sacra, realizzato contro il Temenos Circolare nel tratto antistante la Porta Sud durante la Fase 3 (Motya VIII, IV secolo a.C.).

58 Si vedano per alcuni esempi i tipi 28,40 e 43 della tipologia di M. Sguaitamatti $(1984,13,16,109-111,131$ 132, 136-141, tavv. 15:52; 21:72; 22:76; 23:80; 40:140).

59 Dalla necropoli di Santa Monica a Cartagine proviene una statuetta, attribuibile ad una matrice siceliota della prima metà del V secolo a.C., cui sono state aggiunte le braccia modellate a mano. Tuttavia, la datazione della tomba in cui la statuetta è stata rinvenuta non può risalire oltre la metà del IV secolo a.C. (Picard 1975, 197 198, tav. XLI:fig. 1; Sguaitamatti 1984, 132, nota 8).

60 L'oggetto è stato rinvenuto all'interno della US.1713, lungo il muro del Temenos Circolare, nel tratto antistante l'Edificio C6-C4.

61 Si veda a titolo esemplificativo Higgins 1954,85 , n. 223, tav. 38:223

62 Ad es. Higgins 1954, 379, n. 1417, tav. 196:1417. 
La schematicità con cui è reso il panneggio induce ad ascrivere la terracotta alla serie di personaggi femminili stanti che ancora intorno alla metà del V secolo a.C. sono contraddistinti da caratteristiche arcaizzanti ${ }^{63}$ (fig. 3:d).

\subsection{Il frammento MC.08.118}

In questa terracotta, proveniente dalla Zona C Sud-Ovest ${ }^{64}$, sono riconoscibili due cuscini sovrapposti, attribuibili verosimilmente all'estremità destra di un trono ${ }^{65}$ (fig. 4:a). Le forme arrotondate dei cuscini, il profilo convesso di quello superiore e l'accentuata sporgenza di quello inferiore consentono di individuare confronti puntuali con esemplari raffiguranti figure femminili (con o senza pettorali) assise su un trono con cuscini sovrapposti $^{66}$ (fig. 4:b). Terrecotte simili da Entella ${ }^{67}$, Morgantina ${ }^{68}$, Camarina ${ }^{69}$ e Gela ${ }^{70}$ inducono a datare il frammento MC.08.118 tra la metà e la fine del V secolo a.C., in accordo con il dato stratigrafico.

\subsection{La terracotta MC.06.451}

In questo frammento rinvenuto nella US.1776 della Zona C Sud ${ }^{71}$ è possibile riconoscere un cane seduto sulle zampe posteriori, con la coda ripiegata e caratterizzato da un modellato vigoroso (fig. 4:c). L'animale era assai verosimilmente raffigurato ai piedi di Artemide, come suggerisce il confronto con una terracotta dal Tofet di analoghe dimensioni, raffigurante un cane alla destra della dea, della quale si conservano le porzioni inferiori della veste e dell' $\operatorname{arco}^{72}$ (fig. 4:d). Si tratta di un tipo appartenente all'ampio gruppo cosiddetto della Artemide Sicula ${ }^{73}$, raffigurata in numerosissimi esemplari prodotti tra la fine del V e la prima metà del IV secolo a.C. da diversi centri sicelioti. Del tipo caratterizzato dal cane accovacciato ai piedi della dea sono note a sua volta numerose versioni $^{74}$. L'inquadramento tipologico e il contesto di rinvenimento consentono di proporre

63 Allegro 1990, 130. La sovrapposizione di himation e chiton distingue anche il Tipo 33 (datato alla metà del V secolo a.C.) della tipologia delle statuette con porcellino realizzata da Sguaitamatti (1984, 118-119, tav. 17:59).

64 Il frammento proviene dalla US.2380, situata a sud dell'angolo meridionale del Kothon e attribuibile alla Fase 4 (Motya VII A, c. 470-425 a.C.).

65 Sembra poco probabile che i due cuscini appartenessero ad una kline. Riguardo le terrecotte con figura recumbente su kline o banchettante si vedano: Barra Bagnasco 1971, 151-156; Spagnolo 2000, 192 (entrambi con ampia bibliografia).

66 Sul margine sinistro del cuscino superiore è possibile notare, nella metà anteriore, un innalzamento della superficie dovuto all'originaria presenza del braccio destro della divinità.

67 Onorati 2016, 85-86, T1133, fig. 29:1; 86, T1136b, fig. 29:2.

68 Bell 1981, 14-15, 125, n. 15, tav. 6.

9 Pautasso 1996, 52, 58, 70, n. 81, tav. X:81.

70 De Miro - Fiorentini 1976-1977, 444-445, tav. XXXVIII:2 e 5.

71 Si tratta di uno degli scarichi realizzati contro il Temenos Circolare, nel tratto antistante la Porta Sud, durante la Fase 3 (Motya VIII, IV secolo a.C.).

72 La terracotta, attribuibile alla prima metà del IV secolo a.C., è stata rinvenuta all'interno del pozzo ad imboccatura quadrata (Bevilacqua 1972, 115-116, tav. LXXXIX:2; Ciasca 1992, 147-148; Mammina - Toti 2011, 34, fig. 12).

73 Su tale gruppo e sulle revisioni cronologiche cui è stato sottoposto negli ultimi decenni si vedano Pisani 2008, 58-70; Pautasso 2012, 165, nota 10.

74 Pisani 2008, 59-60 (con estesa bibliografia). 
per il frammento MC.06.451 una datazione tra la fine del V e la prima metà del IV secolo a.C.

\subsection{La terracotta MC.05.20}

L'esemplare è stato rinvenuto nel margine settentrionale del Santuario C $3{ }^{75}$. Anche questa terracotta, caratterizzata da un rilievo poco prominente e dal retro piatto, raffigura uno dei tipi ascrivibili al gruppo della cosiddetta Artemide Sicula (fig. 4:e). La dea, stante e con la gamba sinistra flessa, indossa un chitoniskos legato alla vita, mentre dalla spalla sinistra scende un mantello che copre interamente il braccio lasciando scoperta solo la mano. Il braccio destro, leggermente flesso, si protende in basso verso il muso di un cerbiatto $^{76}$ che lambisce la mano della dea ${ }^{77}$. Sotto la spalla sinistra sono stati incisi, dopo la cottura, due solchi che partendo dal medesimo punto si dirigono con andamento divergente verso il busto della figura.

Fra le numerose versioni che, con dettagli differenti, raffigurano Artemide con un cerbiatto o un cane, la terracotta moziese può essere paragonata ad esemplari rinvenuti a Fontana calda di Butera ${ }^{78}$ e a Morgantina ${ }^{79}$ e trova un confronto puntuale nell'area del Santuario della Malophoros di Selinunte ${ }^{80}$. Il contesto di rinvenimento e i confronti individuati consentono di datare l'esemplare moziese al IV secolo a.C.

\section{CONSIDERAZIONI CONCLUSIVE}

Dopo l'esame analitico delle terrecotte è possibile esprimere alcune considerazioni sulle iconografie, le produzioni, le figure divine e i loro ambiti di azione, senza dimenticare i limiti sottesi a questo genere di riflessioni. Innanzitutto, è bene sottolineare che nello studio della coroplastica votiva non è sempre possibile istituire una corrispondenza diretta tra iconografia di una divinità, attestazione del culto ad essa tributato e titolarità del luogo di culto in cui la terracotta è stata rinvenuta. Secondariamente, occorre ricordare che i reperti qui presi in esame sono stati sovente rinvenuti in stato frammentario e in giacitura secondaria e pertanto non consentono di formulare ipotesi sulle pratiche rituali. Infine, va tenuto presente il ridotto numero di terrecotte rinvenute rispetto all'estensione dell'area

75 La terracotta proviene dalla colmata di argilla verdastra (US.919) con la quale, durante la realizzazione del Santuario C3 (attività 3c) vennero obliterate le rovine del Tempio C2 distrutto nel 397 a.C. (attività 4a).

76 Sembra meno verosimile che si tratti di un cane, sebbene questo animale sia a volte raffigurato in terrecotte simili, come riscontrabile nei confronti citati nelle note seguenti.

77 Lo schema iconografico corrisponde alla Versione 2 individuata da M. Pisani nella disamina delle terrecotte appartenenti al gruppo della cosiddetta Artemide Sicula (Pisani 2008, 60, nota 115). In tale versione, tuttavia, Artemide è affiancata da un cane.

78 Adamesteanu 1958, 655, nn. 70-73, fig. 283 (a destra). Sulla coroplastica votiva da Fontana calda di Butera si veda da ultimo Portale 2008.

79 Bell 1981, 155, n. 204a, tav. 54:204a; l'esemplare, datato al IV secolo a.C., costituisce un buon confronto per l'iconografia della dea, ma non per l'animale alla sua destra che, nel caso di Morgantina, è forse rappresentato da un cane il quale non solleva la testa verso l'alto.

80 Gabrici 1927, 296, tav. LXXVII:8. Si tratta del confronto più pertinente, sia per l'iconografia della dea, sia per la posizione e il gesto dell'animale che tuttavia è definito da Gabrici come un cane. La terracotta proviene da uno degli strati post 409 a.C. ma non è possibile stabilire se essa fu rinvenuta nell'area del cosiddetto megaron della Malophoros o nel recinto di Zeus Meilichios. 
indagata nel corso dei quattro anni di scavo qui presi in considerazione e in confronto ai rinvenimenti dal Tofet. Tale discrepanza potrebbe essere ascrivibile sia ai culti e ai rituali differenti praticati nei due santuari, sia alle dinamiche di rinvenimento ${ }^{81}$ e pertanto le considerazioni che seguono hanno carattere provvisorio. Qualora le ricerche successive confermassero il ridotto numero di terrecotte votive rispetto alle altre classi di reperti rinvenuti nell'Area Sacra del Kothon ${ }^{82}$, occorrerà indagare in modo più approfondito le ragioni di tale dato.

Nonostante tali limiti, appare opportuno confrontare le figure divine raffigurate nelle terrecotte - insieme ai loro attributi e alle loro prerogative - con il quadro delle divinità, degli ambiti cultuali e dei rituali che è possibile ricostruire sulla base delle installazioni dell'Area Sacra del Kothon, e delle altre categorie di reperti. L'obiettivo è quello di provare ad individuare alcune delle possibili motivazioni che portarono alla scelta dei soggetti e delle iconografie attestate e contribuire così alla ricostruzione del panorama cultuale moziese. Prima di tali considerazioni è opportuno ricordare che, allo stato attuale, sono state individuate due figure divine quali titolari dell'Area Sacra del Kothon: Astarte ${ }^{83} \mathrm{e}$ $\mathrm{Baal}^{84}$.

\subsection{Le iconografie e le produzioni}

Da un punto di vista iconografico le terrecotte qui esaminate sono quasi tutte riconducibili ad ambito ellenico, con le sole eccezioni costituite dal frammento MC.07.87 e dalla ruota MC.08.11785. Per otto terrecotte è attestata una produzione non locale, mentre gli esemplari realizzati a Mozia sono quattro. Fra questi, due frammenti (la protome MC.08.246 e il piede di statuetta stante MC.08.225) sono riconducibili a iconografia greca. Tali dati, sebbene basati su un numero ridotto di reperti, possono essere accostati al quadro noto per il Tofet, dove, nonostante la maggioranza delle terrecotte sia di produzione locale per via dell'alto numero di statuette realizzate al tornio, fra le terrecotte da matrice prevalgono in maniera sostanziale quelle di produzione o tipologia greca ${ }^{86}$. La preponderanza di iconografie di area ellenica nell'Area Sacra del Kothon, di tradizione fenicia ${ }^{87}$, non deve stupire poiché se i dati vengono letti in maniera diacronica confermano un quadro già noto per Mozia: fra le terrecotte esaminate gli esemplari più antichi (la protome o maschera MC.07.87 e la ruota MC.08.117) sono di produzione locale e rientrano

81 Nel Tofet sono state rinvenute quasi mille terrecotte durante un decennio di ricerche archeologiche. La maggior parte dei reperti, tuttavia, era raccolta all'interno di tre favisse, frutto di concentrazioni volontarie del materiale votivo (Mammina - Toti 2011, 33; 2018, 140). Le terrecotte dell'Area sacra del Kothon provengono invece da rinvenimenti isolati.

82 Anche nelle campagne di scavo successive a quella del 2008 è stato rinvenuto un numero ridotto di terrecotte. Si veda la bibliografia citata alla nota 5 .

83 Nigro 2019

84 Guizzi 2012; Nigro - Spagnoli 2012, 8-11; Nigro 2018, 256-257, 262-269.

85 Sebbene non sia possibile risalire all'iconografia dell'oggetto cui la ruota apparteneva, la sua produzione locale e la diffusione di questa tipologia di reperti in ambito fenicio, e più in generale levantino, inducono a non annoverare la ruota fra le terrecotte di iconografia ellenica.

86 Amadasi Guzzo 1969, 54; Bevilacqua 1972, 114; Mammina - Toti 2011, 33

87 Sulla tradizione levantina riconoscibile nell'architettura, nella planimetria e nelle installazioni del Tempio del Kothon si veda in particolare: Nigro 2015b, 84-91. 
ancora in tipologie della tradizione levantina o cipriota, mentre a partire dalla seconda metà del VI secolo a.C. ha inizio l'importazione di terrecotte non locali, cui si affiancano in seguito matrici importate e esemplari di iconografia ellenica prodotti localmente ${ }^{88}$, testimonianze dell'acquisizione stabile di tali iconografie da parte della produzione moziese $^{89}$ e del ruolo svolto dall'isola nella loro diffusione in area punica ${ }^{90}$.

$\mathrm{Nel}$ corso del $\mathrm{V}$ e del IV secolo, arco cronologico cui si può ricondurre buona parte delle terrecotte dall'Area Sacra del Kothon, la diffusione di temi e gusti di origine greca a Mozia e più in generale in area punica ha ormai raggiunto livelli profondi e, quanto alla produzione coroplastica, circolano ormai solo iconografie elleniche ${ }^{91}$.

\subsection{Le figure divine e il loro ambito cultuale}

Le iconografie attestate, ad eccezione della protome o maschera MC.07.87 e della ruota MC.08.117, trovano confronti nei principali insediamenti greci di Sicilia e in particolare nel Santuario della Malophoros di Selinunte ${ }^{92}$. All'interno del repertorio esaminato i personaggi individuabili con certezza sono tre: il sileno, Demetra e Artemide.

La figura del sileno sembra avere avuto discreta fortuna nell'isola dove è presente con alcune varianti ${ }^{3}$. Nella disamina delle possibili motivazioni che spieghino la presenza nell'Area Sacra del Kothon della matrice con sileno, è opportuno ricordare l'appartenenza di quest'ultimo al corteggio dionisiaco - al quale in questo caso potrebbe alludere il kantharos - e quindi all'ambito ctonio $^{94}$. All'interno dell'Area Sacra del Kothon, tale associazione era già testimoniata da una coppa a figure rosse con sileno e iscrizione dedicatoria deposta nel rituale di chiusura del pozzo P.1660, a sud dell'angolo meridionale del bacino del Kothon ${ }^{95}$.

88 Bisi 1990, 6-7.

89 La produzione locale di terrecotte mediante matrici importate da area siceliota è ampiamente nota a Mozia (Bisi 1990, 26-27). Per le terrecotte di iconografia greca ma di produzione moziese dal Tofet si veda Mammina - Toti 2011, 33, 35. Per una matrice greca rinvenuta nell'area artigianale: Falsone 1989, 61, fig. 22; Toti 2008, 78, fig. 10.

90 Bisi 1990, 34

91 Sulla diffusione di tradizioni greche nel repertorio vascolare moziese si vedano: Ciasca 1991a, 183-185; Orsingher 2011, 114, 128-129; Giardino 2013.

92 Confronti con esemplari rinvenuti nel santuario della colonia megarese sono stati riscontrati per la protome MC.08.246, il frammento di statuetta con pettorali MC.08.127, il frammento con mano e melagrana MC.08.210, il piede di figura femminile stante MC.08.225 e l'Artemide MC.05.20.

93 Oltre alle diverse attestazioni nella ceramica figurata, alla matrice MC.05.396 e alla terracotta dalla Zona E ( 2.6 e nota 57), sono da ricordare una matrice raffigurante un sileno con tirso, rinvenuta in uno scarico del IV secolo a.C. sulla strada messa in luce nella zona B (Toti 2008, 77-78, fig. 10), e una testa di sileno in bronzo sporadica dall'area nota come Abitato Centrale (Spagnoli 2015).

94 Dioniso, oltre ad essere legato al mondo infero condivide (come dio della vegetazione) la sfera ctonia con Demetra. Diversi apprestamenti cultuali connessi con il sottosuolo erano presenti in questa area sacra: nel Tempio C5 della fine dell'VIII-VII secolo a.C. (Nigro 2010b, 25), nel Tempio C1 della seconda metà del VI secolo a.C. (Nigro 2009b, 83-84; 2012a, 298), nel Tempio C2 del V secolo a.C. (Nigro 2009b, 84-85; 2012a, 299). Da ultimo: Nigro 2018. Uno degli aspetti più importanti di Dioniso, inoltre, è la sua caratterizzazione come divinità dell'elemento umido e della natura rigogliosa. Riguardo a tali caratteristiche, attestate nelle fonti letterarie, si veda Gasparri - Veneri 1986, 414-417.

95 Nigro - Spagnoli 2012, 53, 57, fig. 35, tav. V. 
Inoltre, la sua appartenenza ai trasgressivi cortei dionisiaci sottolinea anche il legame del sileno con i temi dell'erotismo e della fecondità, ben rappresentati nell'Area Sacra del Kothon dal Tempio di Astarte ${ }^{96}$, presso il quale la matrice è stata rinvenuta.

È verosimile, pertanto, che fossero in atto processi di assimilazione o sovrapposizione tra la figura del sileno e quella di Bes, dovuti non solo ad alcune somiglianze fisiche ma anche ad ambiti comuni, fra i quali vanno annoverati proprio quelli dell'erotismo e del vino $^{97}$.

La terracotta MC.08.210, sulla quale è raffigurata una mano con melagrana, attribuibile all'iconografia di Demetra, proviene dal settore situato a sud dell'angolo meridionale del Kothon. In quest'area si trovavano non solo il già menzionato pozzo P.1660, ma anche la favissa F.2950 e il pozzo P. $2927^{98}$, installazioni nelle quali sono stati rinvenuti semi, ossa di suini e di bovini che potrebbero essere ricondotti o a una figura di tipo demetriaco o alla stessa dea greca ${ }^{99}$. In merito a quest'ultima ipotesi, può essere utile ricordare che, secondo un'antica tradizione mitologica localizzata in Arcadia, la dea era legata a Poseidon ${ }^{100}$. A questa divinità, o al suo equivalente fenicio (Baal 'Addir) sono stati attribuiti alcuni dei rinvenimenti dal pozzo P. $2927^{101}$. Tuttavia, nel caso della terracotta in questione, tenuto conto delle titolarità divine individuate nell'Area Sacra del Kothon, più che alla possibilità di un culto di tipo greco tributato alla divinità ellenica - forse difficile da ipotizzare come pienamente praticato a Mozia già nella prima metà del $\mathrm{V}$ secolo a.C. - è possibile pensare $\mathrm{a}$ fenomeni di assimilazione, sovrapposizione o interpretatio tra Demetra e Astarte ${ }^{102}$, la quale poteva anche essere riconosciuta nelle iconografie demetriache dai fedeli di tradizione fenicia e punica.

All'iconografia di Artemide, infine, possono essere attribuiti la terracotta con cane MC.06.451 e la figura femminile MC.05.20. Nel caso del secondo reperto, in particolare, appare suggestiva la possibilità di riconoscere la lettera A (alpha) nelle due linee incise sotto la spalla sinistra della figura femminile, come se il dedicante avesse voluto rendere esplicita l'iniziale del teonimo, corrispondente sia ad Artemide che ad Astarte ${ }^{103}$. Quanto ad Artemide, secondo alcune tradizioni essa sarebbe figlia di Demetra e figurava inoltre

$96 \quad$ Nigro 2019.

97 In tali processi un ruolo determinante era svolto dal sostrato egiziano ancora presente nella cultura moziese, come sottolineato da Spagnoli 2015, 45.

98 Sui rinvenimenti non ceramici da tali installazioni: Nigro - Spagnoli 2012, 33-35, 49-50.

99 Sulla figura di Demetra e sui principali luoghi di culto ad essa dedicati nel Mediterraneo si veda Di Stefano ed. 2008. A una figura di questo tipo potrebbero essere ricondotti alcuni falcetti rinvenuti in diversi settori dell'Area sacra del Kothon (MC.06.32; MC.05.360; Nigro 2011, 7-8).

100 Beschi 1988, 845-846.

101 Nigro - Spagnoli 2012, 50; 2018 (in particolare p. 65).

102 Su questa divinità si veda Bonnet 1996. Per simili fenomeni di assimilazione e interpretatio in ambito etrusco tra Vei e Demetra si veda Belelli 2012, 462-464.

103 La lettera A è riconoscibile, sia in caratteri fenici che greci, in alcuni dei rinvenimenti dall'Area sacra del Kothon: una alfa incisa sul fondo esterno di una kotyle datata alla prima metà del VI secolo a.C. dalla Zona C Nord (MC.06.1737/2); una alef incisa sul fondo esterno di una coppa attribuita alla seconda metà del V secolo a.C. con raffigurazione di sileno (MC.11.4512/2) dal Pozzo P.1660 (Nigro - Spagnoli 2012, 53, fig. 35); ancora un'alfa incisa su un ciottolo ipoteticamente attribuito ad Astarte (Nigro 2010a, 163, nota 5) e rinvenuto sulla banchina orientale del Kothon della Fase 4 (ca. 470-425 a.C.), relativa al Tempio C2 (Nigro ed. 2005 , 128, 200, tav. XXXIII:MC.04.284). 
nell'episodio del ratto di Persefone ${ }^{104}$. La dea cacciatrice veniva localizzata non solo sui monti e nelle foreste ma anche nelle paludi e presso le fonti di acqua dolce ${ }^{105}$, ambienti che ricordano la sorgente del Kothon e, più in generale, le condizioni della Laguna dello Stagnone in cui si trova Mozia ${ }^{106}$. Le fonti letterarie e iconografiche, inoltre, attribuiscono arco e frecce ad Artemide che era appellata anche come "arciera" e "dispensatrice di frecce" 107 . Non si può fare a meno di ricordare, a questo proposito, le punte di freccia in bronzo che, di risulta dagli strati della distruzione del 397 a.C., vennero offerte in alcuni depositi del Santuario C3 nel corso del IV secolo a.C. ${ }^{108}$. Significativo appare, inoltre, il luogo di rinvenimento della terracotta con iconografia di Artemide, in uno dei settori più importanti del Santuario C3 (al di sopra dell'adyton del precedente Tempio C4 del V secolo a.C.), subito ad est di una concentrazione di depositi votivi accomunati dalla presenza di palchi e altre porzioni di cervo, animale sacro alla dea ${ }^{109}$. Inoltre, è opportuno sottolineare che, secondo diverse tradizioni mitiche, Artemide era la compagna di Orione ${ }^{110}$, figura assimilabile al semitico Baal - cui sembrano ricondurre alcune caratteristiche del Tempio del Kothon ${ }^{111}$. Si ricorda, infine, che la seconda delle tre fasi della monetazione moziese (secondo quarto del V secolo a.C.) è caratterizzata dall'emissione di didrammi con i tipi della testa femminile e del cane, raffigurato stante o nell'atto di sbranare una testa di cervo $^{112}$.

104 Kahil 1984, 618; Beschi 1988, 845. La compresenza di Artemide e Demetra all'interno della stessa area sacra è attestata, oltre che nel santuario urbano di Metaponto, in diversi siti in Grecia e Magna Grecia (Calabrìa 2005, 80-81, note 67-68). Sul rapporto tra Artemide e Demetra si veda anche Canopoli 2016, 65-66.

105 Si pensi ad es. alla fonte Aretusa, sacra ad Artemide, presso Siracusa, della quale parla Diodoro V, 3, 5-6.

106 Nigro - Spagnoli 2017, 6-9.

107 Kahil 1984, 618-619.

108 Si tratta in particolare dei depositi D.1576, D.1577, D.1597, D.2185. Sulle categorie di reperti, fra i quali le punte di freccia, che componevano i depositi del Santuario C3 si veda Nigro 2009a, 706-707.

109 Depositi D.72, D.649, D.690, D.691, D.688, D.686 (Nigro ed. 2004, 55; 2005, 75-78). Sui legami tra Artemide e il cervo si veda Bevan 1986, 100-114. Nella vicina Monte Polizzo, i numerosi palchi di cervo rinvenuti nella Zona $\mathrm{A}$ sono stati attribuite al culto di una divinità locale forse assimilata ad Artemide a partire dal V secolo a.C. (Morris et al. 2003, 280-285). Ad una figura di questo tipo potrebbero essere ricondotti anche i resti di cane (altro animale ad essa legato) rinvenuti fra le deposizioni effettuate nella Favissa F.2950 e nel pozzo P.1660 dell'Area Sacra del Kothon (Nigro - Spagnoli 2012, 33). Sui legami tra il cane e Artemide si veda: Bevan 1986, 115-130. Infine, i frammenti di testuggine offerti nel Pozzo P.1660 (Nigro - Spagnoli 2012, 57, fig. 39) potrebbero rimandare ancora ad una figura tipo Artemide; per le raffigurazioni di tartaruga dedicate a questa divinità in diversi santuari della Grecia: Bevan 1986, 159-167; 1988.

110 Alcune fonti raccontano anche che, per cause diverse, la dea uccise il gigante cacciatore, il quale dopo la morte fu tramutato in costellazione insieme al suo cane, la stella Sirio (Brelich 1958, 74-76; Fontenrose 1981 6-7, 12-20). Le ossa di cane rinvenute fra le deposizioni della Favissa F.2950 e del pozzo P.1660 (si veda la nota precedente) potrebbero pertanto essere legate anche a questo personaggio mitico.

111 Sugli elementi riconducibili ad Orione: Nigro 2010c, 21-22; sui reperti attribuiti a Baal: Guizzi 2012; Nigro Spagnoli 2012, 50, 57; Nigro 2018. Sull'assimilazione tra Baal e Orione e sull'importanza di questo personaggio nella tradizione semitica occidentale si veda Lawson Younger 2012.

112 Gli stessi tipi ricorrono in alcune emissioni segestane forse realizzate dai medesimi conii (Cutroni Tusa 1989 177-179; 2004, 491-492). A Segesta, inoltre, in tetradrammi dell'ultimo quarto del V secolo a.C. il cane compare a fianco di un giovane cacciatore nel quale è stato proposto di riconoscere l'eroe fondatore Egeste; per tale interpretazione si veda Marconi 1997, 1071-1087. 
Non si può escludere in questo caso che nel IV secolo a.C. la dea greca, cacciatrice, Potnia Theron e legata anche alla guerra ${ }^{113}$ venisse assimilata o sovrapposta - almeno da alcuni frequentatori dell'area sacra - ad Astarte per via delle caratteristiche comuni alle due dee, fra le quali anche quelle legate alla guerra. Infatti, nonostante in ambito fenicio e punico Astarte non sembra avere avuto caratteristiche da cacciatrice - attestate invece ad Emar e Ugarit - Filone di Biblo accomunava Astarte e Artemide ${ }^{114}$. Inoltre, secondo Cicerone una statua di Artemide venne sottratta dai Cartaginesi a Segesta all'inizio del III secolo a.C. e portata a Cartagine, forse per essere venerata secondo il rito greco o per tributare il culto a una divinità fenicia o punica (Astarte $\mathrm{o}$ Tinnit) dalle caratteristiche molto simili ${ }^{115}$. Ancora una volta, pertanto, è possibile ipotizzare, a seconda del background culturale dei fedeli, scenari diversi e mutevoli nel corso del tempo che comprendono il culto della divinità titolare fenicia Astarte (anche per il tramite di iconografie elleniche) e fenomeni di assimilazione o sovrapposizione tra divinità appartenenti a tradizioni diverse ma con alcune caratteristiche in comune (Astarte e Artemide).

Le riflessioni qui esposte hanno introdotto la complessa questione riguardante le divinità che i frequentatori dell'Area Sacra del Kothon, e più in generale dei luoghi di culto moziesi, riconoscevano nelle terrecotte di iconografia greca. Le diverse possibilità risultanti dalla combinazione tra la tradizione in cui si riconosceva il dedicante, l'ambito iconografico della terracotta e la divinità venerata, possono essere sintetizzate, con riferimento all'Area Sacra del Kothon, in due casistiche principali:

1) il personaggio riconosciuto dal dedicante corrispondeva pienamente a quello originariamente raffigurato nella terracotta. Per fare un esempio concreto, in una terracotta con iconografia di Artemide, sarebbe stata riconosciuta proprio quella divinità da parte di un dedicante di cultura ellenica, che dedicava la terracotta in un luogo di culto fenicio consacrato alla fenicia Astarte per via delle caratteristiche comuni fra le due dee ${ }^{116}$.

2) il dedicante non identificava nella terracotta il personaggio solitamente associato a quella iconografia ma vedeva in essa una figura divina diversa, dotata di caratteristiche e prerogative simili ${ }^{117}$. Nella terracotta con iconografia di Artemide un dedicante di cultura punica avrebbe riconosciuto Astarte. Inoltre, a tale proposito, quando si valutano i fattori che portavano alla scelta di una terracotta di tipo greco per un culto tributato a una divinità

113 Sui legami tra il culto di Artemide in Attica e la guerra si veda Canopoli 2016, 120-124 (con ulteriore bibliografia).

114 Bonnet 1996, 23, 30. Sulla mutevolezza del record archeologico legato al culto di Artemide in Sicilia e Italia meridionale si veda Parisi 2010.

115 Ribichini 2015, 161-163, 165 (con ulteriore bibliografia).

116 Sui fenomeni di assimilazione, sovrapposizione o interpretatio nella religione fenicia: Ribichini 1999, 165169. In particolare, l'aryballos laconico (databile alla metà del VI secolo a.C.) con inscrizione in greco (inquadrabile entro il VI secolo a.C.), rinvenuto nella Favissa F.2950 posta a sud del Kothon, sembra testimoniare una dedica rivolta a Belos, interpretatio greca di Baal (Guizzi 2012, 13-15). Sul tema della presenza di Greci e di culti ellenici a Mozia si veda Ampolo 2012, 25-29 che, sulla scia di Diod. XIV, 53, 2, propende cautamente per l'esistenza di templi greci sull'isola e non per la presenza di luoghi di culto di divinità venerate da Fenici e Greci.

117 Tale possibilità è stata considerata da A.M. Bisi come univoca chiave di lettura per interpretare le statuette di produzione greca rinvenute nel Tofet nelle quali, secondo la studiosa, i moziesi avrebbero visto Astarte e Tanit. Il riconoscimento di figure divine greche si sarebbe verificato solo a partire dal IV secolo a.C., con l'introduzione del culto di Demetra e Kore a Cartagine (Bisi 1990, 35). 
fenicia, occorre ricordare che nel IV secolo a.C. fra i prodotti coroplastici di Mozia circolavano quasi esclusivamente iconografie di origine ellenica. In conclusione, sembra evidente che anche le iconografie greche qui individuate possano essere ricondotte alle divinità fenicie e puniche titolari dell'Area Sacra del Kothon - e in particolare ad Astarte -, confermando i numerosi dati emersi negli ultimi anni e contribuendo all'interpretazione di alcuni dei reperti di questa area sacra.

\section{CAtalogo}

MC.07.87 (fig. 1:a)

Frammento di protome o maschera. Lunghezza conservata: $5,5 \mathrm{~cm}$; larghezza conservata: 2,3 cm; spessore 1,2 cm; peso: 21,7 g. Argilla locale; colore corpo: 2.5YR4/8 (Red); segni di steccatura, paralleli all'asse longitudinale, sulla superficie esterna posteriore; superfici interna ed esterna ricoperte da velatura biancastra (2.5Y8/2 Pale Yellow).

Datazione: metà VIII - primo quarto VII secolo a.C.

Confronti: Ciasca 1991b, 30-31, fig. 10.

MC.08.117 (fig. 1:b)

Ruota fittile. Diametro: 7,5 cm; spessore mass.: 2,1 cm; spessore min.: 0,7 cm; peso: 59,4 g. Argilla locale; colore corpo: 2.5YR5/8 (Red); superfici ricoperte da velatura biancastra; colore velatura: 2.5 Y $8 / 2$ (Pale Yellow).

Datazione: seconda metà VI - prima metà V secolo a.C.

Confronti: Nigro ed. 2005, 206, tav. XXXVI:MC.04.40; Panvini - Sole edd. 2009, 79, n. $\mathrm{VII} / 26$.

MC.08.127 (fig. 1:c)

Frammento di statuetta con pettorali. Altezza conservata: 2,3 cm; larghezza conservata: 2,1 $\mathrm{cm}$; spessore minimo: 0,3 cm; spessore massimo: 0,5 cm: peso: 2,4 g. Argilla non locale, depurata; colore corpo: 10YR7/8 (Yellow); tracce di velatura biancastra sulla superficie esterna; colore velatura: 2.5Y8/2 (Pale Yellow).

Datazione: seconda metà VI - fine V secolo a.C.

Confronti: Dewailly 1992, 47-48, fig. 13.

MC.08.246 (fig. 1:e)

Frammento di protome femminile. Altezza conservata: $6,5 \mathrm{~cm}$; larghezza conservata: 4,3 $\mathrm{cm}$; spessore mass.: 1,7 cm; spessore min.: 1,0 cm; peso: 61,0 g. Argilla locale; colore corpo: 10R5/8 (Red); ampie tracce di velatura biancastra; colore velatura: $2.5 \mathrm{Y} 8 / 2$ (Pale Yellow); segni di steccatura sulla superficie posteriore esterna.

Datazione: prima metà V secolo a.C.

Confronti: Beer 2000, 1249-1250, fig.1. 
MC.08.210 (fig. 2:a)

Frammento di statuetta con mano e melagrana. Altezza conservata: $4,8 \mathrm{~cm}$; larghezza conservata: $5,3 \mathrm{~cm}$; spessore: $1,0 \mathrm{~cm}$; peso: $24,2 \mathrm{~g}$. Argilla non locale; colore corpo: 7.5YR7/4 (Pink); piccole tracce di velatura biancastra sulla superficie esterna; colore velatura: $2.5 \mathrm{Y} 8 / 2$ (Pale Yellow).

Datazione: prima metà V secolo a.C.

Confronti: Gabrici 1927, 273, tav. LX:4.

MC.05.396 (fig. 2:c)

Matrice frammentaria con sileno. Altezza conservata: 7,4 cm; larghezza conservata: $5,9 \mathrm{~cm}$; spessore: $1,4 \mathrm{~cm}$; peso: $75,2 \mathrm{~g}$. Argilla non locale, depurata; colore corpo: 5YR6/8 (Reddish Yellow).

Datazione: prima metà V secolo a.C.

Confronti: Winter 1903a, 218, n. 2.

MC.08.225 (fig. 2:d)

Base con piede. Altezza base: $3,9 \mathrm{~cm}$; altezza massima conservata: $5,4 \mathrm{~cm}$; lunghezza base: $7,1 \mathrm{~cm}$; larghezza conservata base: 4,9 cm; spessore parete base: $0,7 \mathrm{~cm}$; peso: $107,7 \mathrm{~g}$. Argilla locale; colore corpo: 2.5YR5/8 (Red); superfici ricoperte da velatura biancastra; colore velatura: $2.5 \mathrm{Y} 8 / 2$ (Pale Yellow).

Datazione: V secolo a.C.

Confronti: Amadasi Guzzo 1969, 93, n.104, tav. LXVII:7; Tusa 1973b, 52, tav. XXV:1.

MC.08.233 (fig. 3:a)

Testa fittile frammentaria. Altezza conservata: $4,5 \mathrm{~cm}$; larghezza conservata: $2,0 \mathrm{~cm}$; spessore: 0,3 cm; peso: 10,9 cm. Argilla non locale, depurata; colore corpo: 10YR7/8 (Yellow). Numerose tracce di velatura biancastra sulla superficie esterna; colore velatura: 2.5Y8/2 (Pale Yellow). Ricomposta da due frammenti; numerose abrasioni.

Datazione: secondo quarto - fine V secolo a.C.

Confronti: Sguaitamatti 1984, 13, 16, 109-111, 131-132, 136-141, tavv. 15:52; 21:72; $22: 76 ; 23: 80 ; 40: 140$

MC.06.80 (fig. 3:c)

Frammento di peplophoros. Altezza conservata: $4,8 \mathrm{~cm}$; larghezza conservata: $3,0 \mathrm{~cm}$; spessore: $0,9 \mathrm{~cm}$; peso: 17,3 g. Argilla non locale, depurata; colore corpo: al centro 5YR5/2 (Reddish Gray); ai margini 5YR7/4 (Pink); superficie esterna ricoperta da velatura biancastra; colore velatura: 5Y8/2 (Pale Yellow).

Datazione: metà V secolo a.C.

Confronti: Sguaitamatti 1984, 118-119, tav. 17:59. 
MC.08.118 (fig. 4:a)

Frammento di statuetta in trono. Lunghezza conservata: $4,3 \mathrm{~cm}$; larghezza conservata: 2,8 $\mathrm{cm}$; spessore: $1,0 \mathrm{~cm}$; peso: $19,1 \mathrm{~g}$. Argilla non locale, depurata; colore corpo: 5YR7/8 (Reddish Yellow); tracce di velatura biancastra sulla superficie esterna; colore velatura: 2.5Y8/2 (Pale Yellow)

Datazione: metà - fine V secolo a.C.

Confronti: Onorati 2016, 85-86, T1133, fig. 29:1; 86, T1136b, fig. 29:2 ; Bell 1981, 14-15, 125, n. 15, tav. 6; Pautasso 1996, 52, 58, 70, n. 81, tav. X:81; De Miro - Fiorentini 19761977, 444-445, tav. XXXVIII:2 e 5.

MC.06.451 (fig. 4:c)

Statuetta con cane frammentaria. Altezza conservata: $8,2 \mathrm{~cm}$; larghezza conservata: $4,3 \mathrm{~cm}$; spessore: $1,1 \mathrm{~cm}$; peso: $62,5 \mathrm{~g}$. Argilla non locale, depurata; colore corpo: 5YR7/8 (Reddish Yellow); numerose tracce di velatura biancastra sulla superficie esterna; colore velatura: 2.5Y8/2 (Pale Yellow).

Datazione: fine $\mathrm{V}$ - prima metà IV secolo a.C.

Confronti: Bevilacqua 1972, 115-116, tav. LXXXIX:2; Mammina - Toti 2011, 34, fig. 12.

MC.05.20 (fig. 4:e)

Statuetta femminile frammentaria. Altezza conservata: $7,1 \mathrm{~cm}$; larghezza massima: $4,8 \mathrm{~cm}$; spessore: $1,8 \mathrm{~cm}$; peso: $38,3 \mathrm{~g}$. Argilla non locale; colore corpo: 5YR7/4 (Pink); tracce di velatura biancastra evidenti soprattutto sulle pieghe del chitoniskos e del mantello; colore velatura: 2.5Y8/2 (Pale Yellow). Ricomposta da due frammenti.

Datazione: IV secolo a.C.

Confronti: Gabrici 1927, 296, tav. LXXVII:8; Adamesteanu 1958, 655, nn. 70-73, fig. 283 (a destra); Bell 1981, 155, n. 204a, tav. 54:204a.

\section{BIBLIOGRAFIA}

Adamesteanu, D.

1958 Butera. Piano della Fiera, Consi e Fontana Calda: Monumenti Antichi dei Lincei 44 (1958), coll. 205-672.

AlBeRTOCCHI, M.

1999 Note di coroplastica punica: le figure femminili con "collane di semi": M. CASTOLDI (ed.), Koiná. Miscellanea di studi archeologici in onore di Pero Orlandini, Milano 1999, pp. 355-368.

2004 Athana Lindia. Le statuette siceliote con pettorali di età arcaica e classica (Supplementi alla Rivista di Archeologia 28), Roma 2004.

2012 La coroplastica siceliota nella prima metà del V sec. a.C.: M. Albertocchi - A. Pautasso (edd.), Philotechnia. Studi sulla coroplastica della Sicilia greca (Monografie dell'Istituto per i Beni Archeologici e Monumentali 5), Catania 2012, pp. 141-161.

Allegro, N.

1990 Le terracotte figurate: Lo stile severo in Sicilia. Dall'apogeo della tirannide alla prima democrazia. Museo Archeologico Regionale, Palermo, 10 febbraio - 30 settembre 1990 , Palermo 1990, pp. 123-131. 
AMADASi GuZzo, M.G.

1969 Catalogo delle terrecotte: A. CiasCA - M. G. GuZzo AmAdASI - G. MAtThiae ScAndone B. Olivieri Pugliese - A. Cutroni Tusa - V. Tusa (edd.), Mozia - V. Rapporto preliminare della Missione congiunta con la Soprintendenza alle Antichità della Sicilia AMPOLO, C. Occidentale (Studi Semitici 31), Roma 1969, pp. 53-104.

2012 Compresenza di ethne e culture diverse nella Sicilia occidentale. Per una nuova prospettiva storica: F. BERLINZANI (ed.), Convivenze etniche, scontri e contatti di culture in Sicilia e Magna Grecia (Aristonothos 7), Trento 2012, pp. 15-57.

BARRA BAGNASCO, M.

1971 I recumbenti: M. BARRA BAGNASCO - C. SABBIONE - G. BACCI - U. SPIGO - G. Molli Boffa (edd.), Locri Epizefiri I. Ricerche nella zona di Centocamere, Firenze 1971, pp. 151-169.

BEER, C.

2000 Two female terracotta protomai from Motya, Area K (campaign 1991): M.E. AubET - M. Barthélemy (eds.), Actas del IV Congreso Internacional de Estudios Fenicios y Púnicos, Cádiz, 2 al 6 de Octubre de 1995, Cádiz 2000, pp. 1249-1254.

BELELLI, V.

2012 Vei: nome, competenze e particolarità cultuali di una divinità etrusca: V. Nizzo - L. LA Rocca (edd.), Antropologia e archeologia a confronto: Rappresentazioni e pratiche del sacro. Atti dell'incontro internazionale di studi, Roma, Museo Nazionale Preistorico Etnografico "Luigi Pigorini", 20-21 Maggio 2011, Roma 2012, pp. 455-478.

BELL, M

1981 Morgantina Studies I. The Terracottas, Princeton 1981.

BERTESAGO, S.M.

2009 Figurine fittili da Bitalemi (Gela) e dalla Malophoros (Selinunte): appunti per uno studio comparato di alcune classi della coroplastica votiva: C. ANTONETTI - S. DE VIDO (edd.), Temi selinuntini, Pisa 2009, pp. 53-69.

BESCHI, L.

1988 Demeter: Lexicon Iconographicum Mytologie Classicae IV, Zürich - München 1988, pp. 844-892.

BESQues, S.

1982 La representation du Silène au canthare: Revue archéologique 2 (1982), pp. 263-272.

BEVAN, E.

1986 Representations of Animals in Sanctuaries of Artemis and other Olympian Deities (BAR International Series 315), Oxford 1986.

1988 Ancient Deities and Tortoise-Representations in Sanctuaries: The Annual of the British School at Athens 83 (1988), pp. 1-6.

BEVILACQUA, F.

1972 Considerazioni sulle terrecotte a stampo: F. Bevilacqua - A. CiasCa - G. Matthiae SCANdone - S. Moscati - V. TuSA - A. Tusa Cutroni (edd.), Mozia - VII. Rapporto preliminare della Missione congiunta con la Soprintendenza alle Antichità della Sicilia BISI, A.M. Occidentale (Studi Semitici 40), Roma 1972, pp. 113-117.

1968 Influenza della coroplastica siceliota sulla produzione punica: Sicilia Archeologica 3 (1968), pp. 41-44.

1990 Le terrecotte figurate fenicie e puniche in Italia (Itinerari V), Roma 1990.

BONNET, C.

1996 Astarté. Dossier documentaire et perspectives historiques (Collezione di Studi Fenici 37. Contributi alla Storia della Religio Fenicio-Punica 2), Roma 1996. 
BLINKENBERG, C.

$1931 \quad$ Lindos. Fouilles de l'Acropole, 1902-1914, I. Les petites objets, Berlin 1931.

BRELICH, A.

$1958 \quad$ Gli eroi greci: un problema storico-religioso, Roma 1958.

CALABRÌA, E.

2005 Coroplastica votiva dal santuario urbano di Metaponto: nuove attestazioni di culto di età classica ed ellenistica: M.L. NAvA - M. OsanNa (edd.), Lo spazio del rito. Santuari e culti in Italia meridionale tra indigeni e greci. Atti delle giornate di studio, Matera, 28 e 29 giugno 2002 (Siris. Studi e ricerche della scuola di specializzazione in Archeologia di Matera, Supplemento I), Bari 2005, pp. 69-82.

CAnopoli, M.

2016 Il culto di Artemide in Attica. Dinamica rituale, contesti e network (VII sec. a.C. - II/III sec. d.C.) (Tesi di Dottorato, Sapienza Università di Roma, https://iris.uniroma1.it/retrieve/handle/11573/1012716/1359037/Tesi_dottorato_Canopoli. pdf, consultata il 24/12/2021).

\section{CAVAllari, F.S}

1884 Selinunte: Notizie degli Scavi di Antichità 1884, pp. 318-325.

CHERIF, Z.

1997 Terres cuites puniques de Tunisie (Corpus delle antichità fenicie e puniche), Roma 1997. CHIARENZA, N.

2015 Una matrice per terrecotte con sileno dall'area sacra del Kothon a Mozia: Vicino Oriente XIX (2015), pp. 51-64.

CIASCA, A.

1980 Mozia - Note sull'architettura religiosa: M.J. FonTANA - M.T. PIRAINO - F.P. Rizzo (edd.),

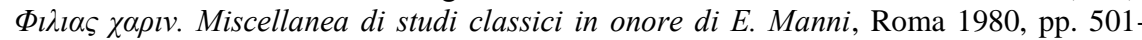
513.

1988 Le protomi e le maschere: S. Moscati (ed.), I Fenici. Catalogo della mostra, Palazzo Grassi, Venezia, 6 marzo - 6 novembre 1988, Milano 1988, pp. 354-369.

1991a La ceramica fenicia di Sicilia e i suoi rapporti con le produzioni coeve: I vasi attici ed altre ceramiche coeve in Sicilia. Atti del convegno internazionale, Catania, Camarina, Gela, Vittoria, 28 marzo - 1 aprile 1990, volume secondo (Cronache di Archeologia 30), Catania 1991, pp. 179-186.

1991b Protomi e maschere puniche (Itinerari VII), Roma 1991.

1992 Mozia: sguardo d'insieme sul Tofet: Vicino Oriente VIII/2 (1992), pp. 113-155.

CiAsCA, A. - TOTI, M.P.

$1994 \quad$ Scavi a Mozia. Le terrecotte figurate (Collezione di Studi Fenici 33), Roma 1994. Cutroni Tusa, A.

1989 La Monetazione dei centri elimi nel corso del V secolo a.C.: G. NENCI - S. TUSA - V. Tusa (edd.), Gli Elimi e l'area elima fino all'inizio della prima guerra punica. Atti del seminario di studi, Palermo - Contessa Entellina, 25 - 28 maggio 1989 (Archivio storico siciliano, Serie 4, vol. 14-15), Palermo 1989, pp. 173-192.

2004 Mozia al centro di un processo: L. NigRo (ed.), Mozia - X. Rapporto preliminare della XXII campagna di scavi - 2002 condotta congiuntamente con il Servizio Beni Archeologici della Soprintendenza Regionale per i Beni Culturali e Ambientali di Trapani (Quaderni di Archeologia Fenicio-Punica, I), Roma 2004, pp. 491-493.

De Miro, E. - Fiorentini, G.

1976-1977 Relazione sull'attività della Soprintendenza alle Antichità di Agrigento (1972-1976): Kokalos 22-23 (1976-1977), pp. 423-455. 
DEWAILLY, M.

1992 Les statuettes aux parures du Sanctuaire de la Malophoros à Sélinonte (Cahiers du Centre Jean Bérard 17), Napoli 1992.

Di STEFANO, C.A. (ed.)

2008 Demetra. La divinità, i santuari, il culto, la leggenda. Atti del I congresso internazionale, Enna, 1-4 luglio 2004 (Biblioteca di «Sicilia Antiqua» 2), Pisa - Roma 2008.

EPIFANIO, E.

1990 La decorazione fittile architettonica: Lo stile severo in Sicilia. Dall'apogeo della tirannide alla prima democrazia. Museo Archeologico Regionale, Palermo, 10 febbraio - 30 settembre 1990, Palermo 1990, pp. 101-105.

FALSONE, G.

1989 Mozia, Zona K. La Quarta campagna di scavo: Sicilia Archeologica 71 (1989), pp. 51-63.

FAMÀ, M.L. - TOTI, M.P.

2000 Materiali dalla "Zona E" dell'abitato di Mozia. Prime considerazioni: Atti delle Terze Giornate Internazionali di Studi sull'Area Elima, Gibellina - Erice - Contessa Entellina, 23-26 ottobre 1997, Pisa - Gibellina 2000, pp. 451-478.

2005 Materiali inediti della Collezione 'G. Whitaker' di Mozia: A. Spanò Giammellaro (ed.), Atti del V Congresso Internazionale di Studi Fenici e Punici, Marsala - Palermo, 2-8 ottobre 2000, Palermo 2005, pp. 615-630.

FAMÀ, M.L. - TOTI, M.P. - VeCCHIO, P.

2002 Terracotte figurate: M.L. FAMÀ, (ed.), Mozia. Gli scavi nella "Zona A” dell'abitato (Collana di Archeologia del Centro Internazionale di Studi Fenici, Punici e Romani, Comune di Marsala 1), Bari 2002, pp. 321-325.

FARISELLI, A.C.

2011 Maschere puniche. Aggiornamenti e riletture iconologiche: Ocnus 19 (2011), pp. 155-170

2014 Maschere antropomorfe in terracotta nell'oriente fenicio: Riflessioni per la redazione di un corpus: A. Lemaire (éd.), Phéniciens d'Orient et d'Occident: Mélanges Josette Elayi (Cahiers de l'Institut du Proche-Orient Ancien du Collège de France II), Paris 2014, pp. 147-167.

FIERTLER, G

2001 La produzione agrigentina di statuette con pettorali: Quaderni di Archeologia 2 (2001), pp. 53-76.

2006 Nuove testimonianze di coroplastica di tipo greco da Mozia: Byrsa V/1-2 (2006), pp. 1127.

FONTENROSE, J.

1981 Orion: The Myth of the Hunter and the Huntress, Berkeley - Los Angeles - London 1981. GABRICI, E.

1927 Il Santuario della Malophoros a Selinunte (Monumenti Antichi dei Lincei, 32), Roma 1927.

GARBATI, G.

2016 Hidden Identities. Observations on the "Grinning" Phoenician Masks of Sardinia: G. GARBATI - T. PEDRAZZI (eds.), "Identity" and Interculturality in the Levant and Phoenician West during the $8^{\text {th }}-5^{\text {th }}$ Centuries BCE (Supplementi alla Rivista di Studi Fenici 42), Roma 2016, pp. 209-228.

GARRAFFO, S.

1990 Tipi monetali di stile severo: Lo stile severo in Sicilia. Dall'apogeo della tirannide alla prima democrazia, Museo Archeologico Regionale, Palermo 10 febbraio - 30 settembre 1990, Palermo 1990, pp. 151-157. 
GASPARRI, V. - VENERI, A.

1986 Dionysos: Lexicon Iconographicum Mytologie Classicae III, Zürich - München 1986, pp. 414-514.

GIARDINO, S.

2013 La ceramica comune tra VI e V secolo a.C. dai recenti scavi a Mozia: dal repertorio originariamente fenicio all'influenza della tradizione greca: L. GIRÓN - M. LAZARICH - M. CONCEIÇÂO (Coords.), Actas del I Congreso Internacional sobre Estudios Cerámicos. Homenaje a la dra. Mercedes Vegas, Cádiz 1 al 5 de noviembre de 2010, Cádiz 2013, pp. 835-859.

GuIzZI, F.

2012 Graffito con dedica votiva su un aryballos dall'area sacra del Kothon a Mozia: L. NiGRo F. Spagnoli (edd.), Alle sorgenti del Kothon. Il rito a Mozia nell'Area sacra di Baal 'Addir - Poseidon. Lo scavo dei pozzi sacri nel Settore C Sud-Ovest (2006-2011) (Quaderni di Archeologia Fenicio-Punica, Colour Monograph 02), Roma 2012, pp. 13-15.

HigGINS, R.A.

1954 Catalogue of the Terracottas in the Department of Greek and Roman Antiquites, British ISSERLIN, B.S.J Museum. Vol. I, Greek: 730-330 B.C., London 1954.

$1971 \quad$ New light on the 'cothon' at Motya: Antiquity 45 (1971), pp. 178-186.

ISSERLIN, B.S.J - COLDSTREAM, J.N. - SNODGRASS, A.

1970 Motya (Trapani). - Rapporto preliminare sugli scavi degli anni 1961-1965: Notizie degli scavi di Antichità 24 (1970), pp. 560-583.

KAHIL, L.

1984 Artemis: Lexicon Iconographicum Mytologie Classicae II, Zürich - München 1984, pp. 618-753.

KARAGEORGHIS, V.

1993 The coroplastic art of Ancient Cyprus, II. Late Cypriote II - Cypro-Geometric III, Nicosia 1993.

1995 The coroplastic art of Ancient Cyprus, IV. The Cypro - Archaic Period. Small male figurines, Nicosia 1995.

1996 The coroplastic art of Ancient Cyprus, VI. The Cypro - Archaic Period. Monsters, animals and miscellanea, Nicosia 1996.

2004 The mask: M.E. AubET (ed.) The Phoenician Cemetery of Tyre-Al Bass. Excavations 1997-1999 (Bulletin d'Archéologie et d'Architecture Libanaises, Hors-Série I), Beyrouth 2004, pp. 414-416.

LAGONA, S.

2003 Una Kourotrophos da Mozia: G. Fiorentini - M. CAltabiano - A. Calderone (edd.), Archeologia del Mediterraneo. Studi in onore di Ernesto De Miro (Bibliotheca Archaeologica, 35), Roma 2003, pp. 435-438.

LAWSON YOUNGER, K.

2012 Another Look at an Aramaic Astral Bowl: Journal of Near Eastern Studies 71,2 (2012), pp. 209-230.

Mammina, G. - Toti, M.P.

2011 Considerazioni sulla coroplastica votiva del tofet di Mozia (Marsala-Italia): Sardinia Corsica et Baleares Antiquae 9 (2011), pp. 31-50. 
2018 Coroplastica votiva "greca" dal tofet di Mozia (Marsala, Italia). Le statuette femminili "Atena Lindia" e "Medma/Locri": M. Guirguis (ed.), From the Mediterranean to the Atlantic: people, goods and ideas between East and West. II. 8th International Congress of Phoenician and Punic Studies, Italy, Sardinia, Carbonia, Sant'Antioco, 21th-26th October 2013 (Folia Phoenicia 2), Pisa - Roma 2018, pp. 140-148.

MARCONI, C

1997 Storie di caccia in Sicilia occidentale: Seconde Giornate Internazionali di Studi sull'Area Elima, Gibellina 22-26 ottobre 1994, vol. II, Pisa 1997, pp. 1071-1120.

MiLLER, R.L.

1983 The terracotta votives from Medma: cult and coroplastic craft in Magna Graecia, Ann Arbor 1983

Morris, I. - Jackman, T. - Blake, E. - Garnand, B. - Tusa, S.

2003 Stanford University excavations on the Acropolis of Monte Polizzo, Sicily, III: preliminary report on the 2002 season: Memoirs of the American Academy in Rome 48 (2003), pp. 243-315.

Moscati, S.

1987 Iocalia punica. La collezione del Museo Nazionale G. A. Sanna di Sassari (Atti della Accademia Nazionale dei Lincei. Classe di Scienze morali, storiche e filologiche. Memorie, Serie VIII, 29), Roma 1987.

NigRO, L.

2009a Offerte e depositi votivi nel Santuario C3 del Kothon di Mozia nel IV secolo a.C.: S. Fortunelli - C. MASSERIA (edd.), Ceramica attica da santuari della Grecia, della Ionia e dell'Italia, Atti Convegno Internazionale Perugia 14-17 marzo 2007, Venosa 2009, pp. 703-719.

2009b Il Tempio del Kothon e le origini fenicie di Mozia: A. MAStino - P.G. SPANO - R. ZucCA (edd.), Naves Plenis Velis Euntes (Tharros Felix 3), Roma 2009, pp. 77-118.

2010a Il Sacello di Astarte e i culti femminili a Mozia: G. BARTOLONI - P. MatTHIAE - L. NigRo L. Romano (edd.), Tiro, Cartagine, Lixus: nuove acquisizioni. Atti del Convegno Internazionale in onore di Maria Giulia Amadasi Guzzo, Roma 24-25 novembre 2008 (Quaderni di Vicino Oriente IV), Roma 2010, pp. 163-180.

2010b Alle origini di Mozia: stratigrafia e ceramica del Tempio del Kothon dall'VIII al VI secolo a.C.: L. Nigro (ed.), Motya and the Phoenician Ceramic Repertoire between the Levant and the West, $9^{\text {th }}-6^{\text {th }}$ Century BC. Proceedings of the International Conference held in Rome, 26 $6^{\text {th }}$ February 2010 (Quaderni di Archeologia Fenicio-Punica V), Roma 2010, pp. 1-48.

2010c L'orientamento astrale del Tempio del Kothon di Mozia: E. AnTONELLo (ed.), Il cielo e l'uomo: problemi e metodi di astronomia culturale. Atti del VII Convegno Nazionale della Società Italiana di Archeoastronomia, Roma, Museo Nazionale Romano, Terme di Diocleziano, 28-29 settembre 2007, Roma 2010, pp. 15-24.

2011 Mozia 2011. Rapporto preliminare sulla XXXI campagna di scavi, prospezioni e restauri dell'Università di Roma "La Sapienza", https://www.yumpu.com/it/document/view/35451438/mozia-2011-missione-archeologicaa-mozia (consultato il 15/09/2021).

2012a The Temple of the Kothon at Mozia, Sicily: Phoenician Religious Architecture from the Levant to the West: M. Gruber - S. Ahituv - G. Lehmann - Z. TAlshir (eds.), All the Wisdom of the East. Studies in Near Eastern Archaeology and History in Honor of Eliezer D. Oren (Orbis Biblicus et Orientalis 255), Fribourg - Göttingen 2012, pp. 293-331. 
2012b Scavi e restauri dell'Università di Roma 'La Sapienza' a Mozia, 2007-2009: il Tempio del Kothon, il Temenos Circolare, il Sacello di Astarte e il Tofet: C. Ampolo (ed.), Agora, foro e istituzioni politiche in Sicilia e nel Mediterraneo antico. Atti delle settime giornate internazionali di studi sull'area elima e la Sicilia occidentale nel contesto del Mediterraneo, Erice 12-15 ottobre 2009, Pisa 2012, pp. 207-218.

2015a Mozia tra VI e V secolo a.C. Monumentalizzazione e organizzazione socio-politica: un nuovo modello: M.P. BAGLIONE - L.M. MichETTI (edd.), Le lamine d'oro a cinquant'anni dalla scoperta. Dati archeologici su Pyrgi nell'epoca di Thefari e Velians e rapporti con altre realtà del Mediterraneo (Scienze dell'Antichità 21.2), Roma 2015, pp. 225-245.

2015b Temples in Motya and their Levantine prototypes: Phoenician religious architectural tradition: A.M. MAILA AfEICHE (ed.), Cult and ritual on the Levantine coast and its impact on the eastern Mediterranean realm: Proceedings of the International Symposium, Beirut 2012 (Bulletin d'Archéologie et d'Architecture Libanaises, Hors-Série X), Beyrouth 2015, pp. 83-108.

2018 La Sapienza a Mozia 2010-2016: il primo insediamento fenicio, l'area sacra di Baal e Astarte, il tofet, la necropoli, l'abitato, i nuovi scavi alle mura - una sintesi: M. GUIRGUIS (ed.), From the Mediterranean to the Atlantic: people, goods and ideas between East and West. II. 8th International Congress of Phoenician and Punic Studies, Italy, Sardinia, Carbonia, Sant'Antioco, 21 ${ }^{\text {th }}-26^{\text {th }}$ October 2013 (Folia Phoenicia 2), Pisa - Roma 2018, pp. 253-277.

2019 The Temple of Astarte "Aglaia" at Motya and its cultural significance in the Mediterranean realm: S. BlaKely - B.J. Collins (eds.), Religious Convergence in the Ancient Mediterranean (Studies in Ancient Mediterranean Religion, 2), Atlanta 2019, pp. 101-125.

NigRO, L. (ed.)

2004 Mozia - X. Rapporto preliminare della XXII campagna di scavi - 2002 condotta congiuntamente con il Servizio Beni Archeologici della Soprintendenza Regionale per $i$ Beni Culturali e Ambientali di Trapani (Quaderni di Archeologia Fenicio-Punica, I), Roma 2004.

2005 Mozia - XI. Il Tempio del Kothon. Rapporto preliminare delle campagne di scavi XXIII e XXIV (2003-2004) condotte congiuntamente con il Servizio Beni Archeologici della Soprintendenza Regionale per $i$ Beni Culturali e Ambientali di Trapani (Quaderni di Archeologia Fenicio-Punica, II), Roma 2005.

2007 Mozia - XII. Zona D. La "Casa del sacello domestico", il "Basamento meridionale" e il Sondaggio stratigrafico I. Rapporto preliminare delle campagne di scavi XXIII e XXIV (2003-2004) condotte congiuntamente con il Servizio Beni Archeologici della Soprintendenza Regionale per $i$ Beni Culturali e Ambientali di Trapani (Quaderni di Archeologia Fenicio-Punica, III), Roma 2007.

NigRo, L. - SPAGNOLI, F.

2012 Alle sorgenti del Kothon. Il rito a Mozia nell'Area sacra di Baal 'Addir - Poseidon. Lo scavo dei pozzi sacri nel Settore C Sud-Ovest (2006-2011) (Quaderni di Archeologia Fenicio-Punica, Colour Monograph 02), Roma 2012.

2017 Landing on Motya. The Earliest Phoenician Settlement of the 8th century BC and the creation of a West Phoenician Cultural Identity in the excavations of Sapienza University of Rome - 2012-2016 (Quaderni di Archeologia Fenicio-Punica/Colour Monograph 04), Roma 2017.

2018 Pomegranate (Punica Granatum L.) from Motya and its deepest oriental roots: Vicino Oriente 22 (2018), pp. 49-90. 
ONORATI, M.T.

2016 La coroplastica: F. Spatafora (ed.), Entella. Il Thesmophorion di Entella. Scavi in ORSINGHER, A. contrada Petraro (Edizioni della Normale, Studi 32), Pisa 2016, pp. 23-100.

2011 La ceramica punica del IV secolo a.C. dalla Fortezza Occidentale: L. Nigro (ed.), Mozia XIII. Zona F. La Porta Ovest e la Fortezza Occidentale. Rapporto preliminare delle campagne di scavi XXIII-XXVII (2003-2007) condotte congiuntamente con il Servizio Beni Archeologici della Soprintendenza Regionale per i Beni Culturali e Ambientali di Trapani (Quaderni di Archeologia Fenicio-Punica, VI), Roma 2011, pp. 112-131.

2014 Listen and protect: Reconsidering the Grinning Masks After a Recent Find from Motya: Vicino Oriente 18 (2014), pp. 145-171.

2018 Across the Middle Sea: The Long Journey of Phoenician and Punic Masks: M. BArbanera (ed.), "La medesima cosa sono Ade e Dionisio": Maschere, Teatro e Rituali Funerari nel Mondo Antico. Atti del Convegno Internazionale, Sapienza Università di Roma, 16-17 novembre 2017 (Scienze dell'Antichità 24, 3), Roma 2018, pp. 51-68.

2019 Across Traditions and beyond Boundaries: The Masks of Carthage: S. BLAKELY - B.J. Collins (eds.), Religious Convergence in the Ancient Mediterranean (Studies in Ancient Mediterranean Religions 2), Atlanta 2019, pp. 295-311.

PANVINI, R. - Sole, L. (edd.)

2009 La Sicilia in età arcaica. Dalle apoikiai al 480 a.C. Catalogo della mostra. Caltanissetta, Museo Archeologico 12 Giugno - 12 Agosto 2006, Catania, Monastero dei Benedettini 26 Ottobre 2006 - 7 Gennaio 2007, Palermo 2009.

PARISI, V.

2010 Offerte votive nei santuari della Magna Grecia: dal contesto archeologico al sistema rituale: M. Dufeu-Muller - S. Huysecom-HaXhi - A. Muller (éd.), Artémis à Épidamne-Dyrrhachion. Une mise en perspective. Table-ronde internazionale, Athènes 19-20 novembre 2010 (Bulletin de Correspondance Hellénique 134), Athens 2010, pp. 454-463.

Pautasso, A.

1996 Terrecotte arcaiche e classiche del Museo Civico di Castello Ursino a Catania (Studi e materiali di archeologica greca, 6), Catania 1996.

2012 L'età classica e tardo-classica. Elementi per un quadro preliminare: M. ALBERTOCCHI - A Pautasso (edd.), Philotechnia. Studi sulla coroplastica della Sicilia greca (Monografie dell'Istituto per i Beni Archeologici e Monumentali 5), Catania 2012, pp. 163-185.

Pelagatti, P.

1965 Antefisse sileniche siceliote: Cronache di Archeologia 4 (1965), pp. 79-98.

2003 Antefisse di provenienza camarinese certa o presunta: G. FioRENTINI - M. CALTABIANO A. Calderone (edd.), Archeologia del Mediterraneo. Studi in onore di Ernesto De Miro (Bibliotheca Archaeologica 35), Roma 2003, pp. 515-532.

PiCARD, C.G.

1967 Sacra punica. Étude sur les masques et rasoirs de Carthage: Karthago XIII (1967), pp. 1115.

1975 Deux thuriféraires de Carthage: Kokalos XXI (1975), pp. 196-204.

PISANI, M.

2008 Camarina. Le terrecotte figurate e la ceramica da una fornace di V e IV secolo A.C. (Studia archeologica, 164), Roma 2008.

Portale, E.C

2008 Coroplastica votiva nella Sicilia di V-III secolo a.C.: La stipe di Fontana Calda a Butera: Sicilia Antiqua 5 (2008), pp. 9-58. 
RIBICHINI, S.

1999 Rileggendo Filone di Biblo. Questioni di sincretismo nei culti fenici: A. MотTE - C. BONNET (éd.), Les syncrétismes réligieux dans le monde méditerranéen antique. Actes du colloque International en l'honneur de Franz Cumont à l'occasion du cinquantième anniversaire de sa mort, Rome Academia Belgica, 25-27 septembre 1997 (Études de Philologie, d'Archéologie et d'Histoire Anciennes 36), Roma-Bruxelles 1999, pp. 149 177.

2015 Statue greche e culti fenici: C. Giuffré Scibona - A. Mastrocinque (edd.), Ex pluribus unum. Studi in onore di Giulia Sfameni Gasparro, Roma 2015, pp. 157-167.

SCHIAVO, D.

$1756 \quad$ Memorie per servire alla storia letteraria di Sicilia. Tomo secondo, Palermo 1756.

SGuaitamatTi, M.

1984 L'offrante de porcelet dans la coroplathie géléenne. Ètude typologique, Mainz am Rhein 1984.

SPAGNOLI, F.

2013 Demetra a Mozia: evidenze dall'area sacra del Kothon nel V secolo a.C.: Vicino Oriente 17 (2013), pp. 153-165.

2015 Una testa di sileno in bronzo da Mozia: Vicino Oriente 19 (2015), pp. 39-50.

2017 Sacrifici e libagioni ad Astarte nell'area sacra del Kothon a Mozia nel V secolo a.C.: E. LiPPOlis - P. VANNICElli - V. PARISI (edd.), Il sacrificio. Forme rituali, linguaggi e strutture sociali (Scienze dell'Antichità 23.3), Roma 2017, pp. 95-107.

SPAGNOLO, G.

2000 Le terrecotte figurate dall'area della stazione vecchia di Gela e i problemi della Toti, M. P. coroplastica geloa nel V sec. a.C.: Quaderni di Archeologia 1/1 (2000), pp. 179-207.

2008 Le «collezioni» del Museo G. Whitaker di Mozia: E. AcQuaro - D. Ferrari (edd.), Le antichità fenicie rivisitate. Miti e culture (Biblioteca di Byrsa, 5), Lugano 2008, pp. 6982.

Tusa, V.

1964 Il Cappiddazzu: A. Ciasca - M. Forte - G. GARbini - S. Moscati - B. Pugliese - V. Tusa (edd.), Mozia - I. Rapporto preliminare della Missione archeologica della Soprintendenza alle Antichità della Sicilia Occidentale e dell'Università di Roma (Studi Semitici 12), Roma 1964, pp. 19-33.

1973a Il Cappiddazzu: A. Ciasca - V. Tusa - M.L. Uberti (edd.), Mozia - VIII. Rapporto preliminare della Missione congiunta con la Soprintendenza alle Antichità della Sicilia Occidentale (Studi Semitici 45), Roma 1973, pp. 7-31.

1973b Il luogo di arsione: A. CIASCA - V. TUSA - M.L. UbERTI (edd.), Mozia - VIII. Rapporto preliminare della Missione congiunta con la Soprintendenza alle Antichità della Sicilia Occidentale (Studi Semitici 45), Roma 1973, pp. 35-56.

1978 La necropoli arcaica e adiacenze. Relazione preliminare degli scavi eseguiti a Mozia negli anni 1972, 1973, 1974: A. CiasCA - G. COACCI Polselli - N. CuOMO Di CAPRIO - M. G. Guzzo Amadasi - G. Matthiae ScAndone - V. Tusa - A. Tusa Cutroni - M. L. Uberti (edd.), Mozia - IX. Rapporto preliminare della Missione congiunta con la Soprintendenza alle Antichità della Sicilia Occidentale (Studi Semitici 50), Roma 1978, pp. 5-90.

UHLENBROCK, J.P.

1988 The Terracotta protomai from Gela: A Discussion of Local Style in Archaic Sicily (Studia Archaeologica, 50), Roma 1988. 
2002 La coroplastica nella Sicilia orientale e meridionale nell'età dei due Dionisi: problemi di stile e cronologia archeologica: N. BonACASA - L. BRACCESI - E. DE Miro (edd.), La Sicilia dei due Dionisi. Atti della settimana di studio, Agrigento 24-28 febbraio 1999, Roma 2002, pp. 321-337.

VAN ROOIJEN, G.

2021 Goddesses of Akragas. A study of terracotta votive figurines from Sicily, Leiden 2021.

VASSALLO, S.

2005 Himera. Città greca. Guida alla storia e ai monumenti, (Beni culturali Palermo: collana di studi e ricerche 8), Palermo 2005.

WIEDERKEHR SCHULER, E.

2004 Les protomés féminines du sanctuaire de la Malophoros à Sélinonte (Cahiers du Centre Jean Bérard, XXII), Napoli 2004.

WINTER, F.

1903a Die antiken Terrakotten. Die Typen der figürlichen Terrakotten, III, 1, Berlin 1903.

1903b Die antiken Terrakotten. Die Typen der figürlichen Terrakotten, III, 2, Berlin 1903.

WHITAKER, J.I.S.

1921 Motya: a Phoenician colony in Sicily, London 1921.

WoYTowitsch, E.

1978 Die Wagen der Bronze- und frühen Eisenzeit in Italien (Prähistorische Bronzefunde, XVII, 1), München 1978. 

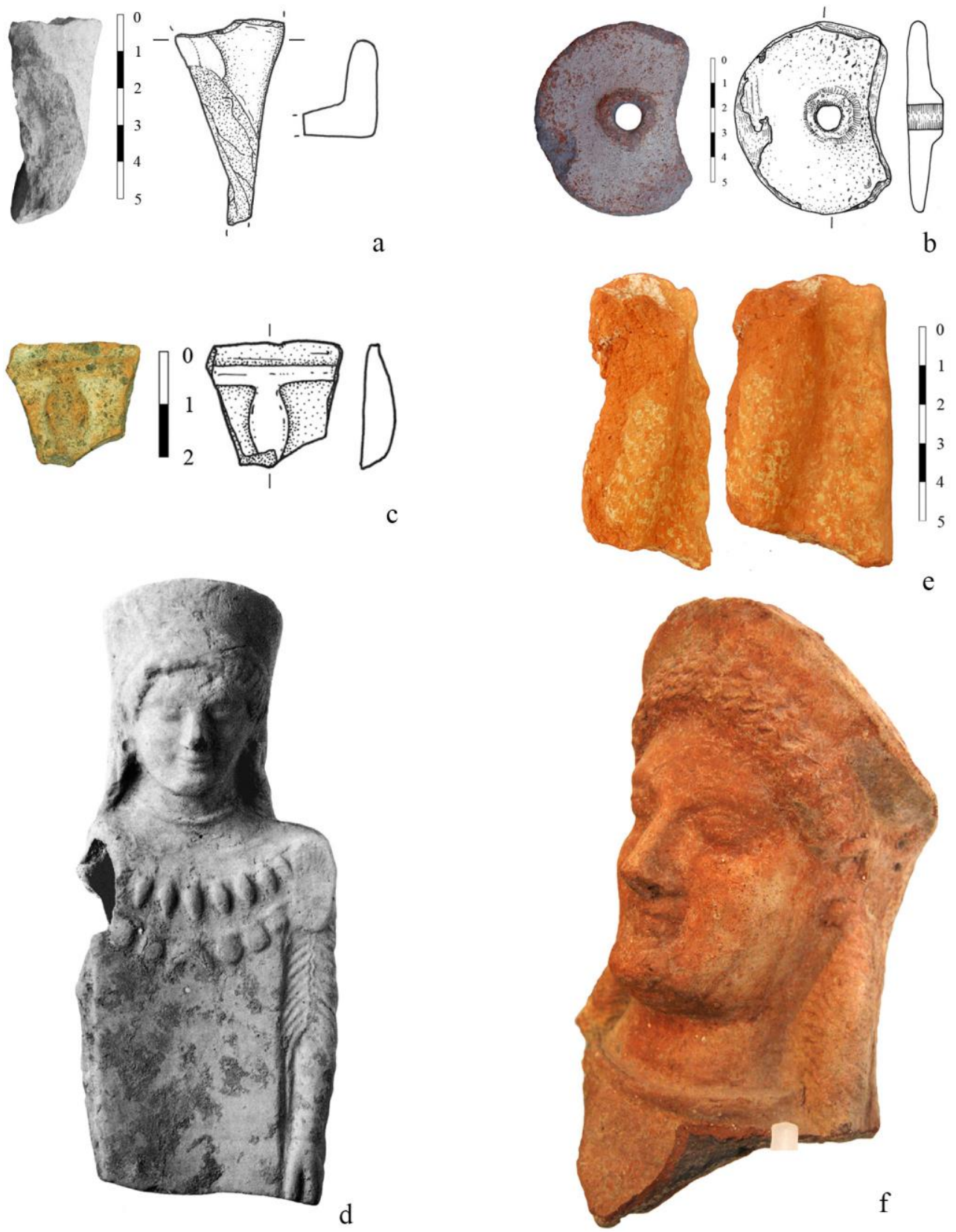

Fig. 1 - a: Frammento di terracotta MC.07.87; b: ruota MC.08.117; c: frammento di statuetta MC.08.127; d: esempio di statuetta con pettorali dal Santuario della Malophoros di Selinunte (Dewailly 1992, 49); e: protome MC.08.246; f: protome femminile M 1005. 




a
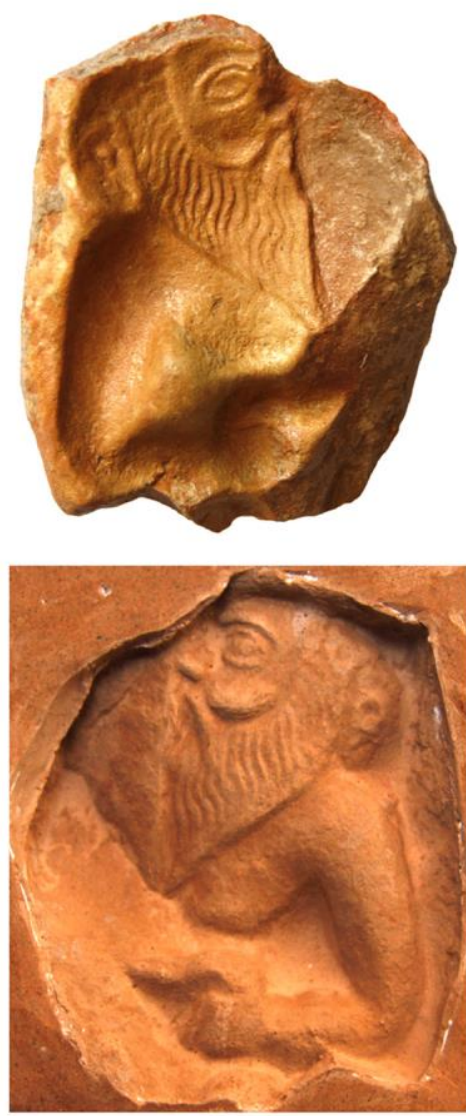

$\mathrm{c}$

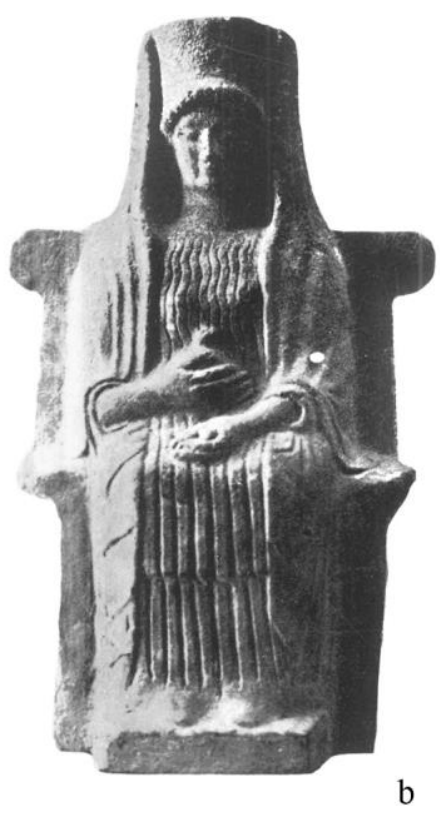

b
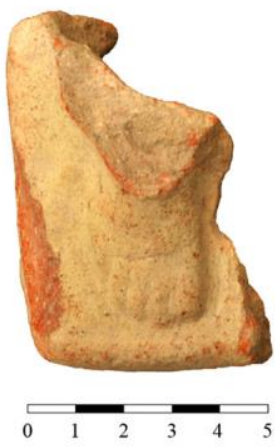

Fig. 2 - a: frammento con melagrana MC.08.210; b: statuetta con melagrana dal Santuario della Malophoros di Selinunte (Gabrici 1927, tav. LX:4); c: matrice MC.05.396 (in alto) e calco da essa ottenuto (in basso); d: base con piede MC.08.225. 

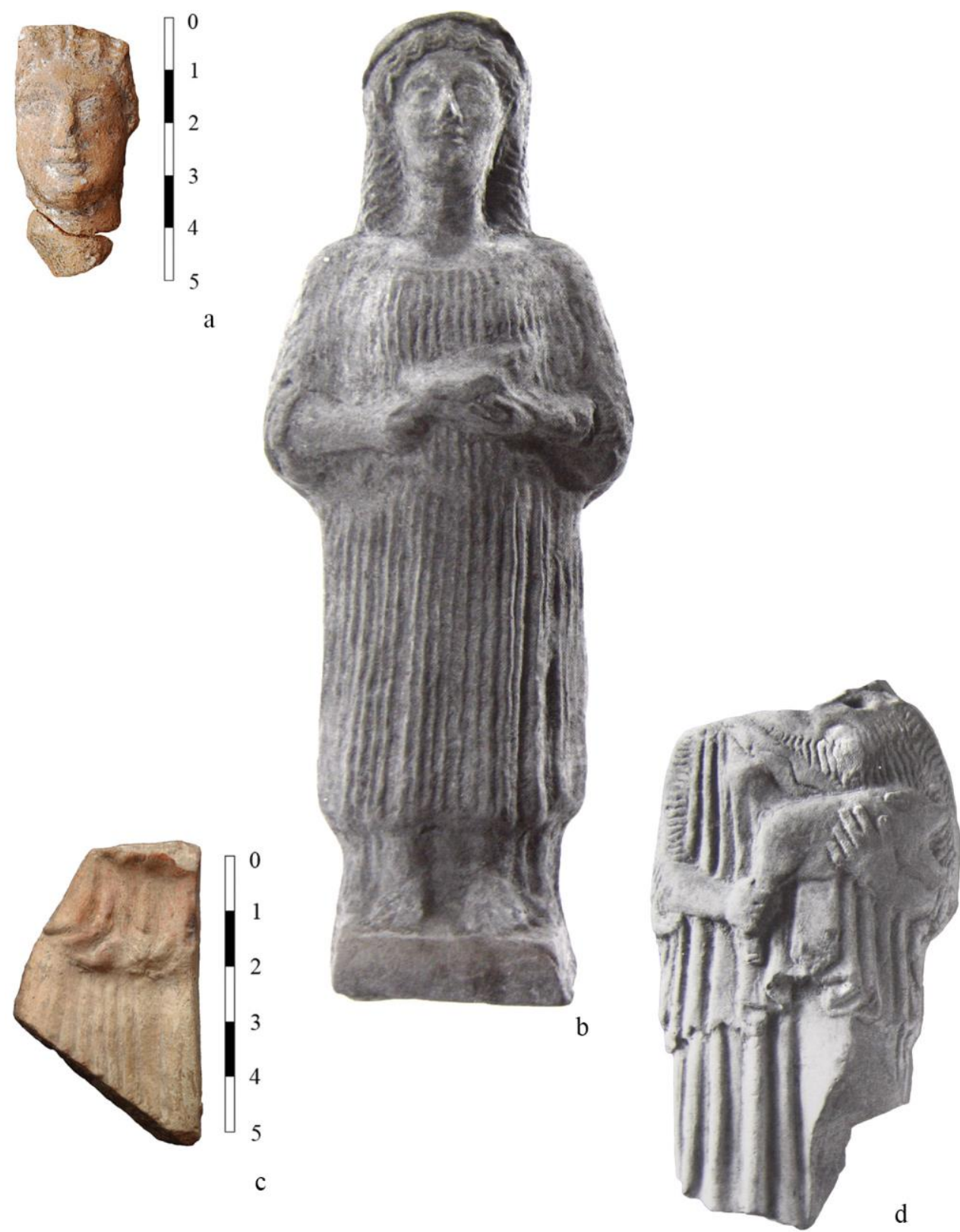

Fig. 3 - a: testa MC.08.223; b: esempio di statuetta di offerente con capigliatura a chevrons (Sguaitamatti 1984, tav. 21:72); c: frammento di statuetta MC.06.80; d: esempio di offerente con himation e chiton (Sguaitamatti 1984, tav. 17:59). 

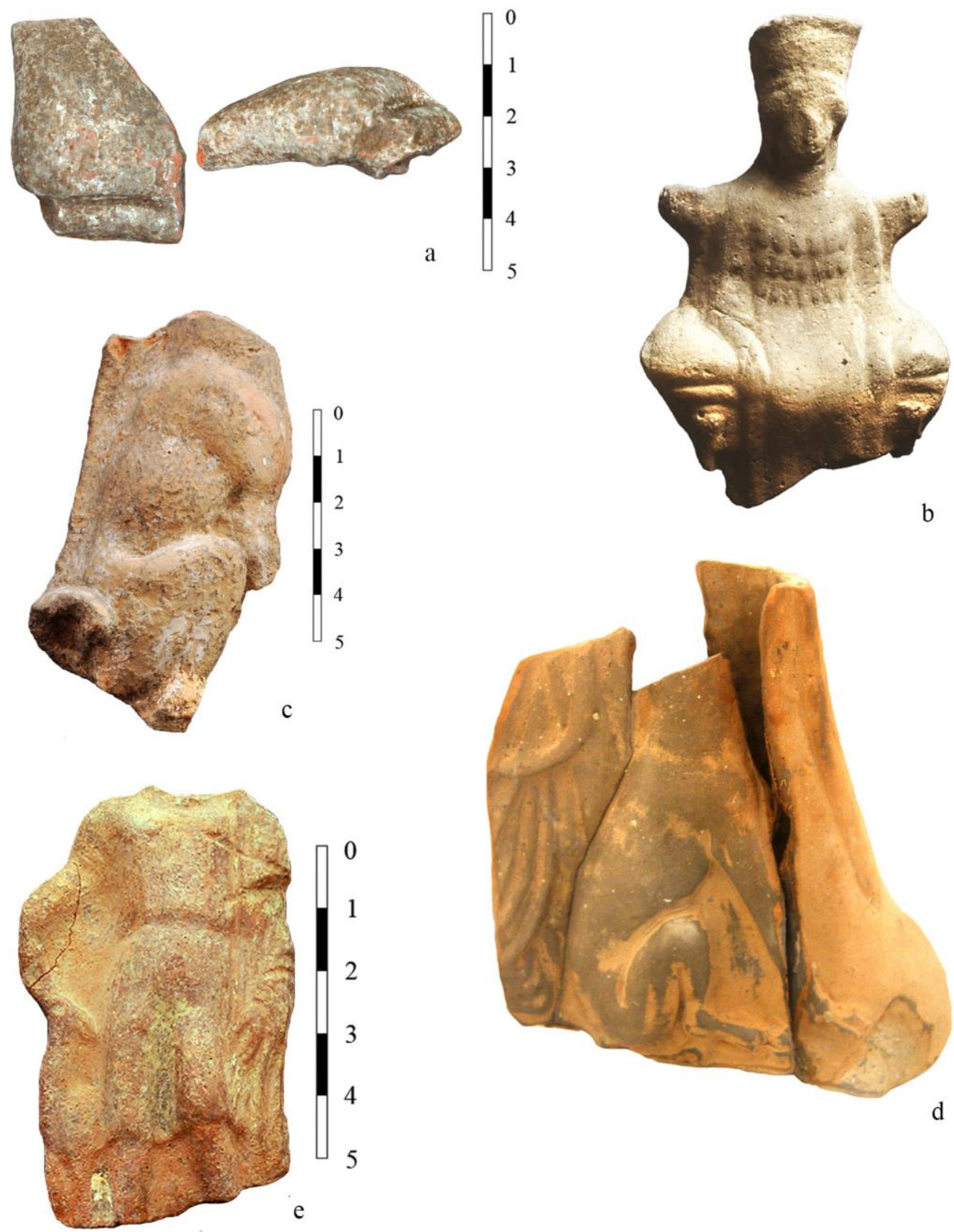

Fig. 4 - a: frammento MC.08.118; b: esempio di statuetta di figura in trono (Onorati 2016, fig. 29:1); c: terracotta MC.08.118; d: terracotta con cane ai piedi di Artemide M 5690; e: terracotta MC.05.20. 